\title{
Minimal-sensing, passive force identification techniques for a composite structural missile component
}

\author{
Nick Stites $^{\mathrm{a}}$, Jonathan White ${ }^{\mathrm{a}}$, Douglas E. Adams ${ }^{\mathrm{a}, *}$ and Matt Triplett ${ }^{\mathrm{b}}$ \\ ${ }^{a}$ Purdue University, Ray W. Herrick Laboratories, 140 S. Intramural Drive, West Lafayette, IN 47906, USA \\ ${ }^{\mathrm{b}}$ U.S. Army RDECOM, AMSRD-AMR-PS-AM, Redstone Arsenal, AL 35898, USA
}

Received 17 September 2007

Revised 2008

\begin{abstract}
Structural health monitoring systems are often limited to the use of one sensor due to cost, complexity, and weight restrictions. Therefore, there is a need to develop load and damage identification techniques that utilize only one sensor. Two passive force estimation techniques are investigated in this work. The techniques focus on either the shape or the amplitude of the magnitude of the applied force in the frequency domain. Both techniques iteratively reduce an underdetermined set of equations of motion into many overdetermined systems of equations to solve for the force estimates. The techniques are shown to locate and quantify impulsive impacts with over $97 \%$ accuracy and non-impulsive impacts with at least $87 \%$ accuracy. A filament-wound rocket motor casing is used as a test structure. Impacts not acting at a specific input degree of freedom are also accurately located depending on the distance away from the modeled input degrees of freedom, and damaging impact forces are quantified by making assumptions about the impulsive nature of the applied force.
\end{abstract}

Keywords: Passive, active, load, force, damage, identification, estimation, quantification, frequency response

\section{Introduction}

Indirect (passive) force estimation, which is the determination of the external forces acting on a structure by measuring only the response of the structure, is a well-studied inverse problem. The benefits of passive force estimation include the ability to warn maintenance crews that a load capable of damaging the structure has occurred, and indirect force estimation can also locate the point of application of the load for further inspection. The difficulty of passive force estimation is that the number of forces acting on a structure often outnumbers the response measurements leading to an underdetermined set of governing equations. An underdetermined set of equations yields non-unique force estimates. However, if certain assumptions about the input forces are valid and the techniques described in this paper are applied, forces on a structure with an underdetermined system of governing equations can be accurately located and quantified.

Two techniques for passive force estimation are thoroughly investigated in this work. The theory of passive force estimation and the two techniques used in this paper are discussed in Section 2. Section 3 includes an analytical example of force estimation for noisy response measurements and for structures that sustain damage. Experimental results are presented in Section 4 where the passive force estimation techniques are used to identify multiple types of impacts (impulsive, damaging, and non-impulsive).

\footnotetext{
${ }^{*}$ Corresponding author. Tel.: +1 765496 8438; Fax: +1 765494 0787; E-mail: deadams@purdue.edu.
} 


\section{Theory}

If any general structure can be modeled as a linear, lumped-parameter system, the $n$ input forces, $F(j \omega)$, and $m$ response measurements, $X(j \omega)$, are related by a matrix $H(j \omega)$ :

$$
X(j \omega)_{m x 1}=H(j \omega)_{m x n} F(j \omega)_{n x 1} .
$$

Each entry in the $X(j \omega)$ vector corresponds to a response measurement from a certain location and in a certain direction on the structure. Similarly, the entries of the $F(j \omega)$ vector correspond to external forces acting at (in) specified locations (directions) on the structure. The frequency response function (FRF) matrix, $H(j \omega)$, describes how the structural properties transform the input forces into output responses.

In passive structural health monitoring (SHM) techniques, the FRFs must be known a priori in order to infer forces given the structural responses. Typically, the $H(j \omega)$ matrix is experimentally estimated (trained) in a test with known input forces and responses, for example, using impact testing, while a specimen is in its baseline (healthy) state. It is important to train the FRF model while the structure is subject to typical operating conditions and environmental loads so that the normal operating and boundary-condition forces are included in the data-driven model of the structure, $H(j \omega)$. By testing under the proper conditions, errors in the estimated external forces can be minimized.

If the number of input forces, $n$, is equal to the number of output responses, $m$, the forces acting on a structure can be estimated by inverting the FRF matrix as follows:

$$
\left(H(j \omega)^{-1}\right)_{n x m} X(j \omega)_{m x 1}=F^{\prime}(j \omega)_{n x 1}
$$

where the prime in $F^{\prime}(j \omega)$ indicates that the forces are indirectly estimated, not measured. If the number of output responses is greater than the number of forces being estimated $(m>n)$, the system of equations is overdetermined, and if the number of outputs is less than the number of inputs $(m<n)$, the system of equations is underdetermined. For an overdetermined (or underdetermined) system of equations, a least-squares, pseudo-inverse technique is often used to estimate the forces $[1,2]$ :

$$
F_{n x 1}^{\prime}=\left(\left[H^{T} H\right]^{-1} H^{T}\right)_{n x m} X_{m \times 1}
$$

where the frequency indices have been excluded for convenience.

Equations (2) and (3) are used to indirectly estimate the forces acting on a structure in the frequency domain, but many other methods estimate the forces in the time domain [3-7]. This paper indirectly estimates forces in the frequency domain because of the ease of implementation of Eqs (2) and (3) and the wealth of reference material from previous investigations of indirect force estimation in the frequency domain [8-14]. Previous work has shown that an overdetermined system of equations in Eq. (3) leads to better numerical stability for the inversion of $H(j \omega)$ than a fully determined $(m=n)$ system [15]. This stability is advantageous because the FRF matrix is often ill-conditioned [7]. When inverting an ill-conditioned FRF matrix, slight perturbations in the response measurements, like measurement noise typically contained in experimental data, are amplified into large, erroneous fluctuations in the force estimates. An overdetermined, least-squares, pseudo-inverse approach for matrix inversion helps minimize these large fluctuations in the force estimates without implementing a regularization technique as is often required when solving force-estimation problems [4,16,17]. Although much literature has been published regarding indirect force estimation, none of the reviewed articles discussed estimating the forces acting on a structure with an underdetermined system of equations with only one passive sensor as is done in this paper

The governing equations of motion for all real structures are inherently underdetermined (when modeled as lumped-parameter systems). Structures have continuous system properties with an infinite number of locations at which input forces can act. As a result, an infinite number of response measurements are required in order to ensure that the forces estimated in Eq. (1) are unique. However, response data often consists of a relatively small number of primary modes of vibration. In these cases, the equations for force estimation can be conditioned to provide reasonable estimates if assumptions can be made about the applied forces and one or both of the techniques described in this paper are applied.

This paper also addresses the technical issues of performing passive force estimation for forces acting on structures that have noisy response measurements or sustain damage that alters the values of the FRF matrix. The necessity 
of "training" the FRF matrix hinders the capabilities of passive health monitoring after the onset of damage because the baseline FRF matrix no longer accurately models the structure after damage occurs. If the input forces are indirectly estimated using the inaccurate $H(j \omega)$ or with noisy response measurements, errors are introduced in the force values. However, if certain assumptions about the input forces can be made, e.g. only one force acts on the structure and that force is broadband impulsive, the force estimates can be curve-fit in the time or frequency domain in order to reduce errors and predict the actual forcing functions.

\subsection{Force estimation by shape}

The techniques for force estimation investigated in this paper are used to estimate non-damaging impulsive forces, damaging impulsive forces, and non-damaging impulsive forces. Both the shape-based technique and the amplitudebased technique discussed in the next section incorporate the aforementioned assumptions (e.g., only one force acts on the structure at one input location and that force is broadband impulsive). The main steps of the new shape-based technique are outlined below:

1. A continuous structure must be discretized such that a finite number of possible force locations are considered. The FRF matrix must be populated by measuring the structural responses for an input force at each discrete location.

2. After an impact, each of the possible force locations is analyzed individually as if that location was the only possible force location. Assuming that more than one response measurement is available, Eq. (1) is solved as an overdetermined inverse problem for every possible forcing location on the structure.

3. The main lobe of the magnitude of the Fourier transform of an impulsive force is broad in frequency and has a smooth roll off with increasing frequency. Because the impact force acting at one of the possible forcing locations is assumed to be impulsive, only the correct force estimate will possess this smooth, roll-off characteristic.

4. A quadratic line is curve fit to each of the force estimates in the frequency domain, and the squared deviation of each force is calculated from its respective curve-fit line and is normalized by the mean of the force magnitude. This normalization emphasizes small deviations in small force estimates and de-emphasizes smaller deviations in large amplitude forces.

5. The force with the lowest normalized squared deviation from its curve-fit line is assumed to be the impact location.

6. The force estimate will most likely not exactly match the actual force because of changes in the component due to various factors including damage that the component may have sustained. Therefore, the force is curve-fit in the time domain to eliminate errors from signal processing, measurement noise, and ill-conditioning of the FRF matrix. The curve fitting is based on the assumption that the impact force is impulsive; consequently, the force is set to zero before and after the peak force with respect to time when the force magnitude falls below a threshold equal to a percentage of the maximum force value. The curve-fitting process is detailed in Section 3.2 of this paper.

\subsection{Force estimation by amplitude}

The second technique of indirect force estimation is also a new technique but is based on the amplitude of the possible force estimates in the frequency domain, rather than the shape of the force estimates as in the previous technique. The main steps of this technique are outlined below:

1. As in the shape-based technique, the structure being monitored must be discretized into a finite number of input DOF, and the FRF matrix must be measured.

2. Using all of the response measurements available, the input forces are estimated at two of the possible force locations. For force estimation utilizing a triaxial accelerometer, which provides three equations of motion, the estimation of two forces is an overdetermined problem. 
3. The values of the magnitude of the two forces are compared in spectral regions near resonant frequencies. Spectral bands far from resonant frequencies and near anti-resonances are extremely susceptible to slight changes in the structural properties and operating conditions because of poor signal to noise ratios, and this susceptibility often leads to erroneous force estimates. In regions near resonant frequencies, the signal to noise ratio is much more advantageous leading to more accurate force estimates. However, as mentioned previously, the FRF matrix is often ill-conditioned near resonant frequencies causing large, incorrect estimates of the forces. This paper demonstrates that the ill-conditioned FRF effects are less disturbing to the force estimates than the signal to noise ratio, and when two forces are compared, the force estimates are often more accurate near resonant frequencies.

4. The ill-conditioned effects when comparing the values of two force estimates are minimized by finding the median of each force over the combined regions of consideration (those near resonant frequencies). The median of the force estimates is not skewed by the large fluctuations that can occur because of the inversion of the FRF matrix. Of the two forces being compared, the force with the highest median value is assumed to be the correct location of the impact force. Neither of the two force locations being considered need be the correct location, but the force that is considered the "correct" force in this step will later be compared to another "correct" force stemming from another pair of possible force locations. Eventually, the force estimate for the location that is actually correct will appear in the final two possible forcing locations and prevail with the highest median value (in theory, the other force to which the correct force is being compared should have amplitudes of zero in the frequency domain because the force only acts at one location).

5. Steps 1-4 are repeated for a new pair of possible input locations until all possible input forces have been compared to at least one other possible force. After this step is completed, the number of possible input locations should have been reduced by at least half.

6. Steps 1-5 are repeated for the remaining possible input forces in order to reduce the number of possible input locations by half again.

7. Steps 1-6 are repeated until only two possible input locations remain. The last two possible forces are compared as steps 1-4 describe in order to identify the location at which the force actually occurred.

8. Using all of the structural responses available, the overdetermined inverse problem of Eq. (3) is solved in order to estimate the force at the location identified in step 7.

9. The estimated force is then curve fit as described in step 6 of the shape-based method in order to find a better estimate of the actual forcing function.

The amplitude-based technique for force estimation appears more complicated and computationally expensive than the shape-based technique. However, the amplitude-based technique actually uses the same number of force estimation calculations as the shape-based method and only uses simple logical intermediate steps to narrow the number of possible forcing locations. The benefit of utilizing the amplitude-based technique with more intermediate steps is realized when identifying and estimating non-impulsive impacts as Section 4.2 describes.

\section{Analytical application}

Consider the linear, discrete four-degree-of-freedom (4-DOF) system shown in Fig. 1. The displacements of the first three masses are measured, emulating the response measurements from a triaxial accelerometer that will be used in the experimental application section, but the displacement of the fourth mass is not. However, it is possible that an input force acts on any of the four masses. The system of equations in Eq. (1) is, therefore, underdetermined. The FRF matrix used in Eq. (1) is populated with respect to a healthy, baseline structure with the properties shown in Table 1. The properties were chosen so that the structural system is lightly damped and so that the modes of vibration are largely uncoupled as in the test specimen used in the experimental section.

A half-sine-wave, impulsive force with a peak amplitude of $150 \mathrm{~N}$ and width of 0.0011 seconds is applied to the fourth DOF and the two aforementioned techniques for passive force estimation are used to estimate the location and magnitude of the force. The force is applied to the fourth DOF rather than any of the first three DOF because in an actual application, such as the rocket motor casing, the impact location is most often not collocated with any response measurements. The impact is assumed to cause damage to the system, and noise is included on the response measurements. The analytical simulation uses a sampling frequency of $10,000 \mathrm{~Hz}$ in order to capture the shape of the input force and a time span of 6.5536 seconds (65536 samples). 
Table 1

System properties and natural frequencies of the 4-DOF shown Fig. 1

\begin{tabular}{llllll}
\hline $\mathrm{m}_{1}$ & $0.25 \mathrm{~kg}$ & $\mathrm{k}_{3}$ & $900,000 \mathrm{~N} / \mathrm{m}$ & $\mathrm{c}_{4}$ & $2.5 \mathrm{~N} / \mathrm{m} / \mathrm{sec}$ \\
$\mathrm{m}_{2}$ & $0.25 \mathrm{~kg}$ & $\mathrm{k}_{4}$ & $300,000 \mathrm{~N} / \mathrm{m}$ & $\mathrm{c}_{5}$ & $2.5 \mathrm{~N} / \mathrm{m} / \mathrm{sec}$ \\
$\mathrm{m}_{3}$ & $0.25 \mathrm{~kg}$ & $\mathrm{k}_{5}$ & $50,000 \mathrm{~N} / \mathrm{m}$ & $\mathrm{f}_{n 1}$ & $49 \mathrm{~Hz}$ \\
$\mathrm{~m}_{4}$ & $0.25 \mathrm{~kg}$ & $\mathrm{c}_{1}$ & $2.5 \mathrm{~N} / \mathrm{m} / \mathrm{sec}$ & $\mathrm{f}_{n 2}$ & $160 \mathrm{~Hz}$ \\
$\mathrm{k}_{1}$ & $50,000 \mathrm{~N} / \mathrm{m}$ & $\mathrm{c}_{2}$ & $2.5 \mathrm{~N} / \mathrm{m} / \mathrm{sec}$ & $\mathrm{f}_{n 3}$ & $233 \mathrm{~Hz}$ \\
$\mathrm{k}_{2}$ & $200,000 \mathrm{~N} / \mathrm{m}$ & $\mathrm{c}_{3}$ & $2.5 \mathrm{~N} / \mathrm{m} / \mathrm{sec}$ & $\mathrm{f}_{n 4}$ & $461 \mathrm{~Hz}$ \\
\hline
\end{tabular}

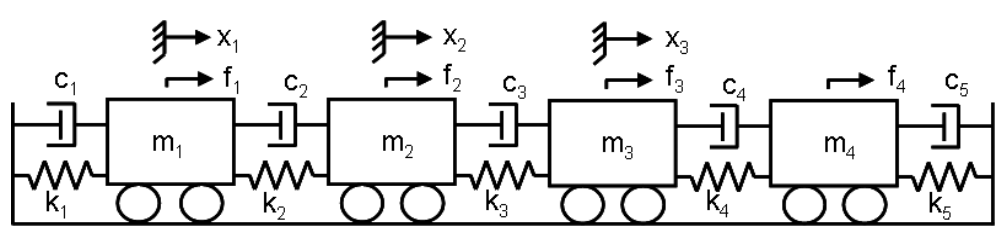

Fig. 1. Four-degree-of-freedom model with underdetermined system of equations of motion.

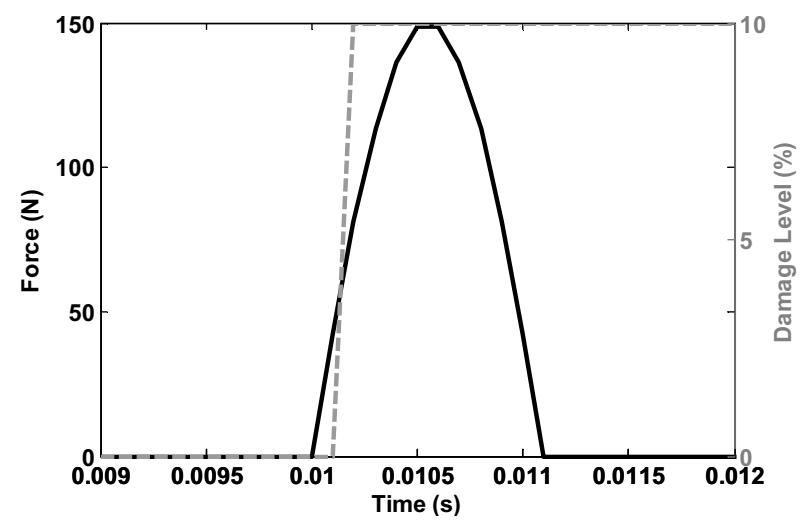

Fig. 2. Relationship between the applied force (- - ) and the damage level (-- - ) of $k_{4}$ for the 4-DOF model.

\subsection{Locating the force}

Consider the 4-DOF structure in Fig. 1 where the fourth mass is subjected to the impulsive force shown in Fig. 2. Suppose that any force equal to or greater than $50 \mathrm{~N}$ causes structural damage to the 4-DOF system in the form of a $10 \%$ stiffness reduction of $k_{4}$. Figure 2 pictorially illustrates the damage level of the model as the impulsive force is applied. During the impact event, the structure sustains damage that alters the material properties and, therefore, the values of the functions in the FRF matrix. Figure 3 illustrates how a $10 \%$ reduction in the stiffness of $k_{4}$ changes the FRF of the first DOF for a force applied at the fourth DOF.

The resonant frequency at approximately $233 \mathrm{~Hz}$ shifts to a lower frequency as a result of the damage and is the mode of vibration that is the most affected by the damage. The shift in frequency of the third mode is significant in understanding errors that are introduced during the passive, force estimation process discussed later in this section. Because the 4-DOF system sustains damage shortly after the force is applied to the fourth DOF, the damaged FRFs govern the response of the system, and, for simplicity, it is reasonable to assume that the system is damaged before the force is applied.

The force applied to the fourth DOF is corrupted by noise forcing functions (e.g. environmental acoustics), which result in noisy response measurements. The random noise forces are also applied to the other three DOF because the noise sources are assumed to be ambient input forces that act on all DOF. The random forces have a mean of zero, standard deviation of one, and a maximum peak-to-peak amplitude of $1.0 \%$ of the root-mean-squared value of the pristine forcing function applied to the fourth DOF. The maximum amplitude of the noise forces is small compared to the maximum amplitude of the applied force because, in application, when an impulsive force capable 

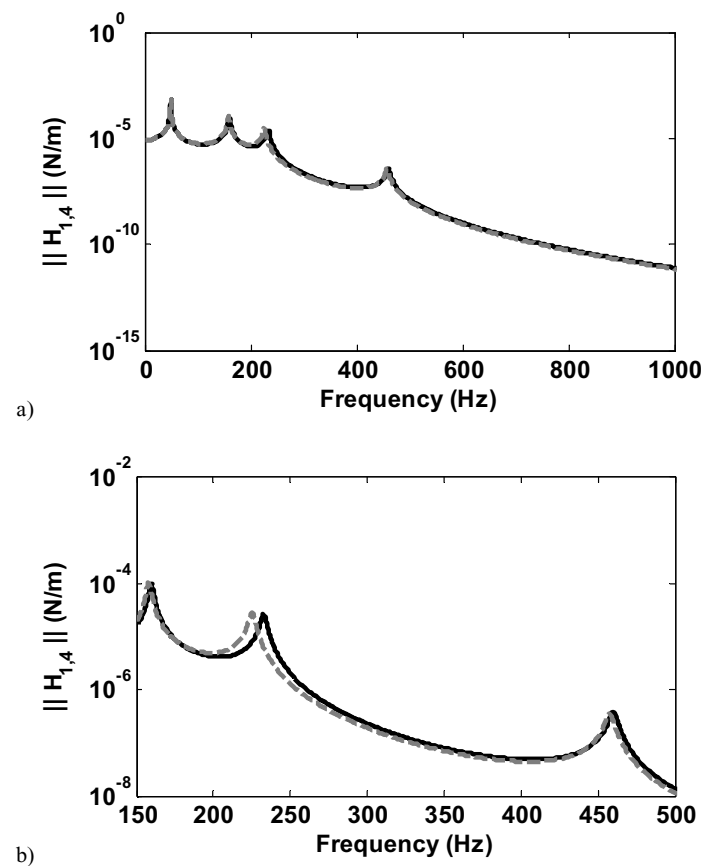

Fig. 3. Comparison of the healthy, trained FRF ( - ) and the damaged FRF (-- $)$ for a) a broad frequency range and b) a narrow frequency range.
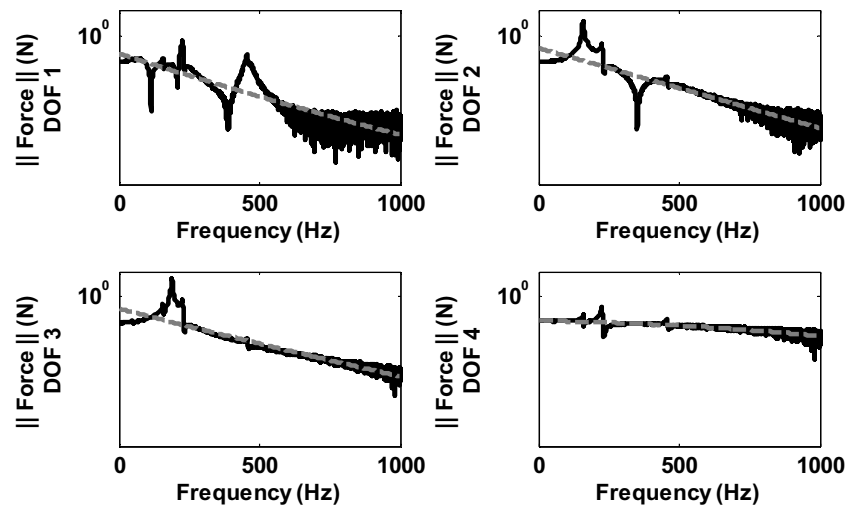

Fig. 4. Force estimates (- and their curve-fit lines (---) for the shaped based technique used to identify a damaging impact on the 4-DOF model.

of producing damage is applied to a structure, the maximum amplitude of the damaging force will be much greater than the amplitudes of random noise forces.

The noisy response measurements for the damaging impact applied to the four DOF differ from the pristine and healthy measurements at frequencies that had the largest change in the FRFs, namely near the third mode of vibration at $233 \mathrm{~Hz}$. Therefore, it is conceivable that the passive force estimates for a damaging impact will have the highest error near frequencies that experience the largest change in the FRFs when damage is sustained. The erroneous force estimates near modes more affected by damage is evident in Fig. 4, especially in the force estimate for the fourth DOF. Figure 4 displays the results of the shape-based method of passive force estimation for an impact on a damaged structure with noisy response measurements.

The location of the force is visually evident by comparing the force estimates of Fig. 4 to their respective curve-fit lines. The force with the lowest deviation from the curve-fit line is that of the fourth DOF, and the force estimate is 

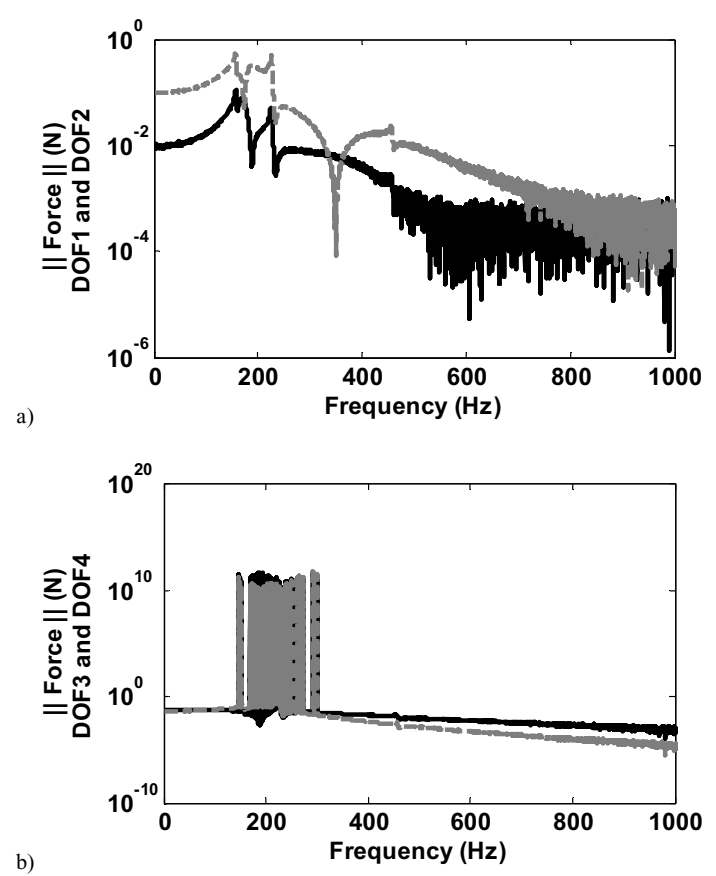

Fig. 5. Force estimates using the amplitude-based technique for a) the first ( - ) and second $(---)$ DOF and b) the third ( - ) and fourth $\mathrm{DOF}(---)$.

already as accurate as possible because all three response measurements were included in the calculation to make the system of equations as overdetermined as possible. The adverse effects of the damage and noisy measurements on the force estimate in the time domain will be discussed after investigating the amplitude-based technique for force estimation.

In order to locate the applied force using the amplitude-based technique for force estimation, the force estimates for the first and second DOF are compared along with the forces for the third and fourth DOF as shown in Fig. 5. The tendency of ill-conditioned FRF matrices to amplify small perturbations in the response data into large, erroneous fluctuations in the force estimates is evident in the force estimates for the third and fourth DOF as displayed in Fig. 5. The large fluctuation in the force estimates for the third and fourth DOF can be explained by analyzing the condition number of the FRF matrix used at each discrete frequency to estimate the forces.

The condition number is a measure of the numerical stability of a set of equations and bounds the possible solution. A matrix with a low condition number (a condition number must be greater than or equal to one) is considered to be well-conditioned and will yield force estimates that closely match the actual forces, and a matrix with a high condition number is ill-conditioned and can yield force estimates that are not close to the actual forces. Roggenkamp showed that any errors in the force estimates must be less than or equal to the errors in the response measurements multiplied by the condition number of the FRF matrix [2,12]. Therefore, FRF matrices with low condition numbers are desirable. Figure 6 shows the condition number of the FRF matrices used in the force estimation calculations for the first and second DOF pair and the third and fourth DOF pair. It is evident in Fig. 5b that the compounded conditions of noisy response measurements and ill-conditioned FRF matrices cause very large, rapid changes in the force estimates for the third and fourth DOF at certain frequencies.

Despite the large inaccuracies of the force estimates for the third and fourth DOF, the median force value for the two sets of force estimates are found in order to determine on which mass within each pair the force most likely acted. Four frequency ranges, each with a span of $20 \mathrm{~Hz}$ and centered around each of the natural frequencies listed in Table 1, are considered when finding the median value. Although the FRF matrix for frequencies near resonant frequencies can have higher condition numbers, it is found that the effect from the ill-conditioned FRF affects the accuracy of the force estimate for frequencies near resonant frequencies less than the SNRs for frequencies between the resonances. Regions between resonances, including antiresonances of FRFs not shown, are more affected by the 
Table 2

Comparison of median force values in the frequency domain for the first iteration of force estimates for a damaging force

\begin{tabular}{cc}
\hline Degree of freedom & Median force value $(\mathrm{N})$ \\
\hline 1 & 0.0104 \\
2 & 0.1033 \\
3 & 0.0247 \\
4 & 0.0474 \\
\hline
\end{tabular}

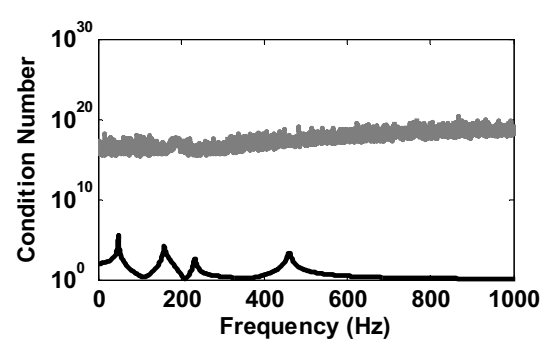

Fig. 6. Condition number for the FRF matrices used in the force estimate calculations for DOF1 and DOF2 ( --$)$ and DOF3 and DOF4 (---).

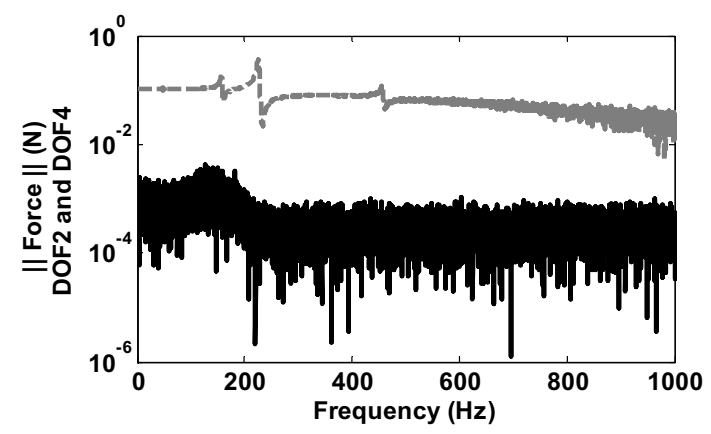

Fig. 7. Comparison of the force at DOF $2(-)$ and DOF $4(---)$ for a damaging impact.

noise than resonant regions because of low SNRs and, therefore, are not considered when finding the median force value. The median values, Table 2, for the four forces suggest that the impact occurred at the second or the fourth DOF, and the force estimates for this pairing are shown in Fig. 7.

Qualitatively, the median force for the force acting on the fourth DOF is much larger than that for the second DOF indicating that the amplitude-based technique correctly identified the force location in the presence of damage and noisy response measurements. The force is quantified as well as possible by repeating the calculation of Eq. (3) using all response measurements to estimate only one force. The result of the calculation is the same as that found for the shape-based technique shown in Fig. 4 for the fourth DOF. The estimate of the applied force is shown in the time domain in Fig. 8.

\subsection{Quantifying the force}

The damage and noisy response measurements cause the force estimate to be incorrect in its peak force estimate and have a ringing, or oscillating, effect after the main lobe of the forcing function. Both errors are primarily the result of damage because simulations involving only noisy measurements and no damage did not produce errors in the force estimates that were as large as in the case of damage as shown in Fig. 8. The damage causes a portion of the signal energy of the force to leak from the main lobe of the forcing function into the subsequent oscillations. The error in the peak force is approximately $7 \%$, but if this error is ignored temporarily, the force estimate at other points 

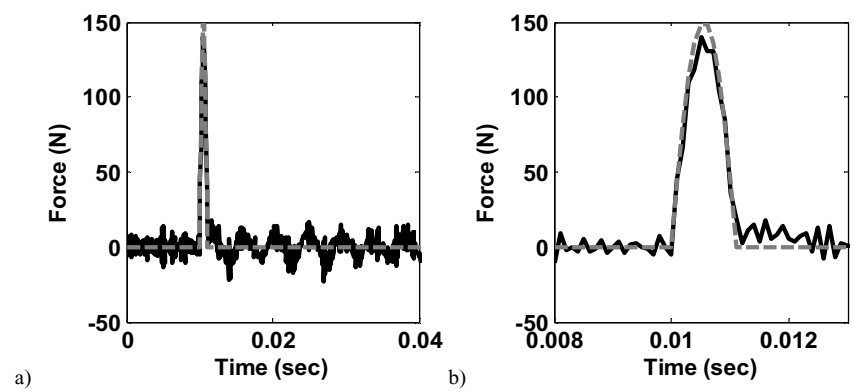

Fig. 8. a) Comparison of force estimate (- $(-)$ and the pristine, actual force (- - ) for a damaging impact; b) close up of main portion of impact force.

in time can be improved if the assumption that the force is impulsive, i.e., similar to the half-sine-wave force of this example, can be made.

If the impact force is impulsive, the applied force is assumed to be zero before the force begins to rise to its peak value, and the force returns to zero soon after the peak value is achieved. By knowing the form of the forcing function in the time domain, the force estimate is curve-fit to minimize the adverse effects from sustained damage and measurement noise. The force estimate is assumed to be zero for all time before the forcing function exceeds a predetermined threshold, and the estimate is assumed to be zero for all time after the peak force when the force estimate drops below the same threshold. This threshold depends on the amount of measurement noise that is typical of the equipment used to collect data and is empirically determined. If the response measurements are very noisy so that the force estimate is also noisy, the threshold for which all force values are neglected and set to zero must be higher than the force estimates with little noise. Also, care must be taken when determining the cutoff threshold because the threshold needs to be large enough to eliminate incorrect force estimates like that in Fig. 8 following the main portion of the forcing function, but not so high as to prematurely constrain the force to zero.

For the analytical example of this section where damage and noise are both included in the force estimate, the threshold below which the force estimate is constrained to be zero is $15 \mathrm{~N}$, or approximately $10 \%$ of the peak force. When this constraint is enforced, the force estimate in both the time and frequency domains is as shown in Fig. 9.

The curve-fit force estimate in Fig. 9 in both the time and frequency domains closely resembles the shape of the applied force that is being passively identified. The large fluctuations of the original force estimate in the frequency domain are absent in the curve-fit force. The curve-fit estimate still has a lower peak amplitude than the actual forcing function, and the Fourier transform coefficients of the curve-fit estimate are also lower than those of the actual force. The Fourier transform coefficients for the curve-fit force are also lower than the original, non-curve-fit force estimate, which is especially noticeable at frequencies below approximately $175 \mathrm{~Hz}$, because some of the energy of the original force estimate is lost due to the curve-fit process when the force is constrained to zero before and after the main portion of the force during the curve-fitting process. In order to conserve energy during the curve-fit process, the curve-fit Fourier transform coefficients need to be adjusted so that the original force estimate and the curve-fit estimate contain the same amount of energy from a signal-based perspective.

The process of equating the energies of the two signals is complicated by the fact that the response measurements contain noise that modifies the energy of the original force estimate. Only the energy in frequencies of the signal that result from the actual impact force rather than the noise should be equated. In frequency regions where the SNR is high, like near the first three modal frequencies, the measurement noise has little contribution in determining the force estimate. Therefore, the energy contained in the signal for frequencies that are less affected by measurement noise are compared and equated between the original and curve-fit estimates.

The total energy of a signal is related to the continuous Fourier transform coefficients by the following equation:

$$
\text { Energy }=|F(0)|^{2}+2 \sum_{\omega=0^{+}}^{\omega=2 \pi f s / 2}|F(j \omega)|^{2}
$$

where $f_{s}$ is the sampling frequency in Hertz, $\omega$ is the circular frequency in radians/second, and the symmetric relationship of $F(j \omega)$ is utilized. Equation (4) must be modified for use with discrete Fourier transform (DFT) 

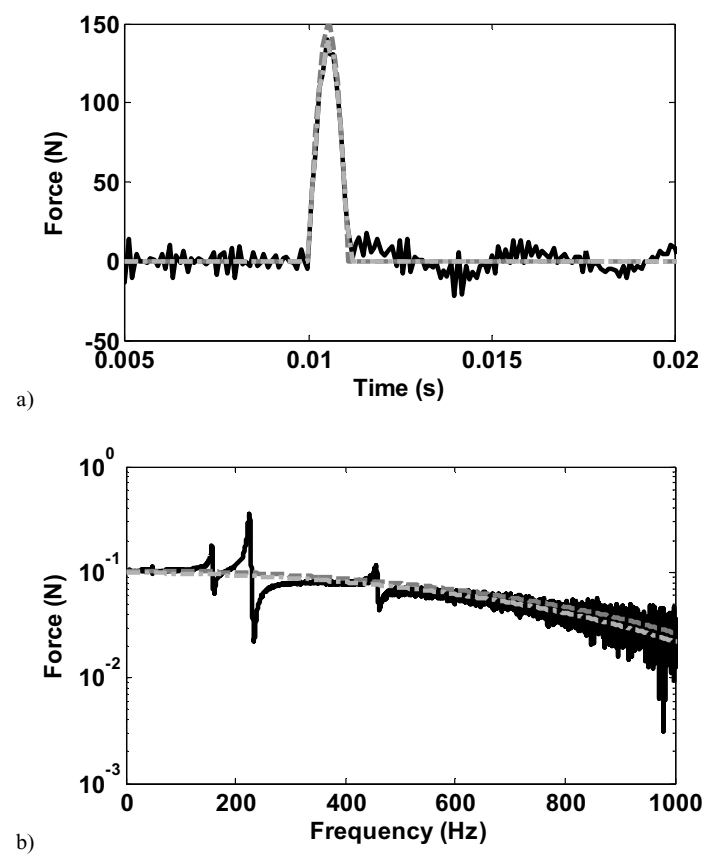

Fig. 9. Comparison of the original estimated force (- $(-)$, the actual force $(---)$, and the curve-fit force $(-\cdot-\cdot)$ in the a) time domain and b) frequency domain.

coefficients because force values at only discrete frequencies are known. The relationship between the continuous Fourier transform coefficients and the DFT coefficients can be approximated for periodic functions by:

$$
F(j \omega) \approx \Delta t F\left(\frac{k}{T}\right)
$$

where $k$ is an index that ranges between zero and the total number of frequencies at which the DFT is computed (denoted as $N$ in later equations), $T$ is the length of time (period) for which the response measurements are recorded, and $\Delta t$ is the time increment between two data samples in the time domain. After substituting Eq. (5) into Eq. (4), the following expression for the total energy of a signal is found:

$$
\text { Energy }=\frac{\Delta t^{2}}{T}\left(|F(0)|^{2}+2 \sum_{k=1}^{k=N / 2}\left|F\left(\frac{k}{T}\right)\right|^{2}\right) .
$$

where $1 / T$ stems from converting a discrete energy spectral density to a discrete energy spectrum.

In order to only consider the energy in the original force estimate due to the actual impact and not measurement noise, only the energy of the signal in frequency ranges with a large SNR is analyzed. Consider the original force estimate compared to the actual force for a frequency range from zero to half the sampling rate as shown in Fig. 10. The noise has a significant effect at higher frequencies as discussed previously, but the shape of the estimated force follows that of the actual force for frequencies up to about $1000 \mathrm{~Hz}$. If an assumption is made that the majority of the signal energy for the force estimate is at frequencies greater than zero (DC offset neglected) and less than $1000 \mathrm{~Hz}$ and is unaffected by noise, only the total energy of the original estimate for frequencies greater than zero and less than $1000 \mathrm{~Hz}$ is compared to the energy of the curve-fit force for similar frequencies:

$$
\text { Scale }=\frac{\sqrt{\frac{\text { Energy Original_Estimate }_{\text {Energy }}}{\text { Curve-fit_Estimate }}}}{2} .
$$

Half of the square root of the quotient of the energy of the two signals in the focused frequency span, Eq. (7), is a scalar number by which the corresponding Fourier transform coefficients are multiplied. The $1 / 2$ factor of Equation (7) accounts for the symmetry of the Fourier transform because only the positive frequencies were considered in the 


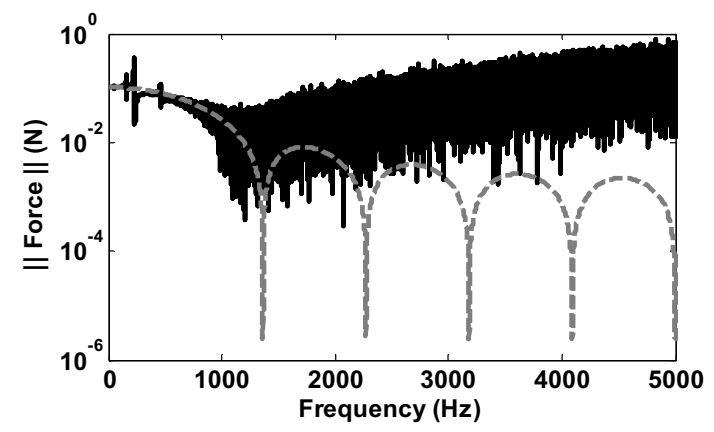

Fig. 10. Comparison of the original force estimate (- ${ }_{-}$) and the actual force $(---)$for a frequency range from zero to half the sampling rate.
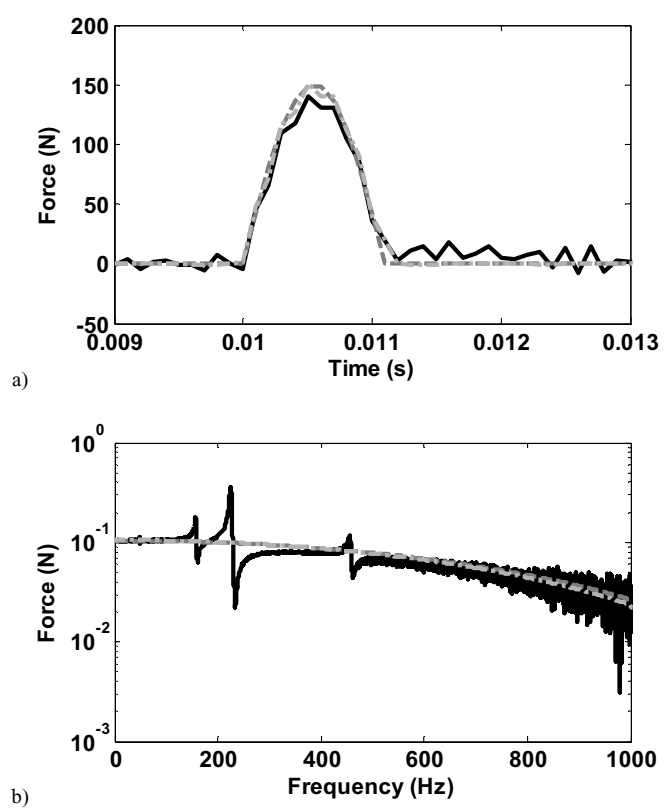

Fig. 11. The original force estimate (- $(-)$, the actual force $(---)$, and the new curve-fit force estimate $(-\cdot-\cdot)$ in the a) time and b) frequency domains.

above analysis. The curve-fit force estimate after its Fourier transform coefficients are adjusted to account for energy that is lost in the curve-fitting process is shown in Fig. 11. It is evident that the peak force increases as well as the DFT coefficients, and the adjusted, curve-fit estimate better matches the actual forcing function. The new curve-fit DFT coefficients also better match the original, non-curve-fit force estimate coefficients at frequencies unaffected by the damage, e.g., for frequencies less than $175 \mathrm{~Hz}$. One adverse effect stemming from the energy adjustment process is that the new curve-fit force estimate has slight oscillations before and after the main lobe of the force in the time domain because the DFT coefficients no longer match a forcing function in which the force is equal to exactly zero before and after the impact. However, the oscillations have very small amplitudes (less than $2 \mathrm{~N}$ peak-to-peak), so the small oscillations contain a very small amount of the total energy of the signal and are neglected (but not eliminated).

The curve-fitting and energy-adjustment processes are crucial steps in finding a force estimate that accurately describes the actual force for a damaged structure and noisy response measurements. The concepts and techniques explained in this section describing the analytical application of the two proposed force estimation techniques are applied experimentally for forces acting on a filament wound rocket-motor casing in the next section. The experiments include locating and quantifying both non-damaging and damaging, impulsive impacts that include 
measurement noise. The experimental tests also investigate the robustness of the two force estimation techniques by attempting to identify non-impulsive impact forces and impulsive forces not acting at a trained DOF.

\section{Experimental application}

A filament-wound rocket motor casing is used as a test structure to investigate the applicability of the two aforementioned passive force identification techniques. The missile casing is approximately 0.178 meters in inner diameter, 0.61 meters in total length, and 7.95 millimeters in thickness. The rocket motor casing is empty for all experimental tests. A single, miniature, triaxial accelerometer (PCB 356A22) is mounted on the nose of the missile casing to acquire three orthogonal acceleration measurements. The sensor is positioned on the nose of the casing because in this location the sensor would be protected inside the warhead instrument panel of the missile. A PCB 086C03 modal impact hammer or Instron Dynatup 9250HV drop tower is used to strike the structure depending on whether low-energy or high-energy impacts, respectively, are investigated. The data from the load cell of the hammer or the load cell in the tip of the drop tower carriage (the tup) is used for comparison to gauge the accuracy of the force estimates. The test setups for each type of test conducted will be further explained in the subsequent sections.

\subsection{Impulsive impacts}

This section investigates the ability of the two techniques for passive force estimation to correctly identify and quantify impulsive forces, similar to the force applied in the previous analytical section, acting on a filament-wound rocket motor casing. As in the analytical application, only isolated impacts are considered, i.e., no concurrent forces are applied to the test specimen. Also, as required by the first step of both force estimation techniques, the missile casing is discretized into 24 possible forcing DOF. Although the number of possible forcing DOF for this application is chosen to be 24, the accuracy of the estimated force does not depend on the number of possible input DOF, i.e., the accuracy of the applied force would be the same (assuming that only three response measurements are available and that the correct input DOF is located) for a missile casing discretized into 18, 24, or 30 points [18]. The accuracy of the force estimate is a function of the ill-conditioned nature of the FRF matrix (assuming that the response measurement is not corrupted), which largely depends on the number of response measurements, not the number of possible input DOF. The number of possible input DOF, when implementing the iterative force estimation techniques presented in this paper, is reduced to one regardless of how many or few possible input DOF there are originally. Alternatively, the precision of the estimated forcing location depends on the number of input DOF using the training model because more input DOF corresponds to less distance between possible input DOF and a more precise estimate of the forcing location. The number of DOF is chosen to be 24 for this application so that the distance between adjacent forcing points is approximately constant. The 24 points are distributed equally among six equidistant rings along the axis of symmetry of the missile casing, and the applied force is assumed to act at one of the 24 locations. Figure 12 shows the discretized rocket motor casing with a triaxial accelerometer used to measure the dynamic structural response.

One triaxial accelerometer, PCB 356A22, is superglued on the conic section, or the nose, of the rocket motor casing (RMC) at a location $25.4 \mathrm{~mm}$ from the metallic insert and in line with the column of impact locations that includes Points 1-6. The accelerometer senses the response of the structure due to forces applied at one of the 24 possible impact locations. As a result of utilizing an accelerometer for recording the structural responses, the FRFs and response measurements are in acceleration units ("g's"), not displacement as in the analytical section. However, because both the FRFs and the responses are in terms of acceleration, no unit conversions or process alterations are needed in either estimation technique.

For the experimental tests discussed in this section, the missile casing is suspended by two rubber bands threaded through two diametric holes at the open end of the canister. The FRF matrix is experimentally populated via modal impact testing. A modal impact hammer is used to hit ten times at each of the 24 locations and the accelerometer responses for each hit are recorded. The FRF for every impact location with respect to the three measurements of the accelerometer are estimated using the well-known $\mathrm{H}_{1}$ estimator [19]. 


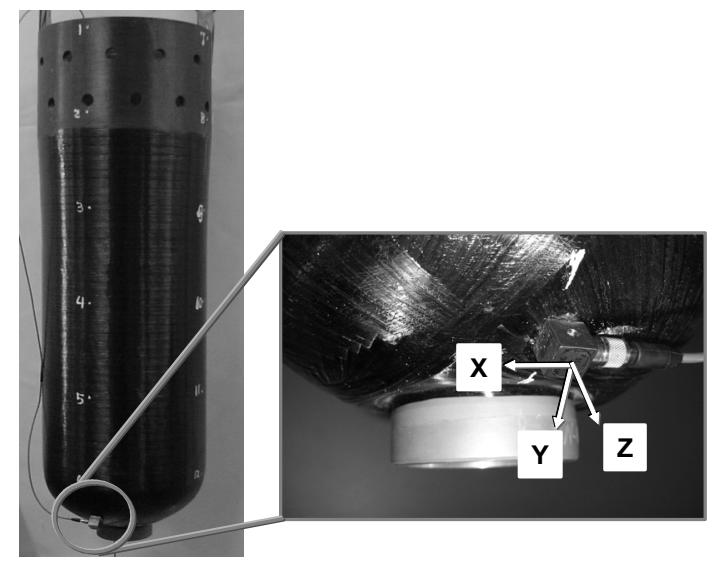

Fig. 12. Filament-wound rocket motor casing that is discretized into 24 possible forcing locations and has a triaxial accelerometer attached.

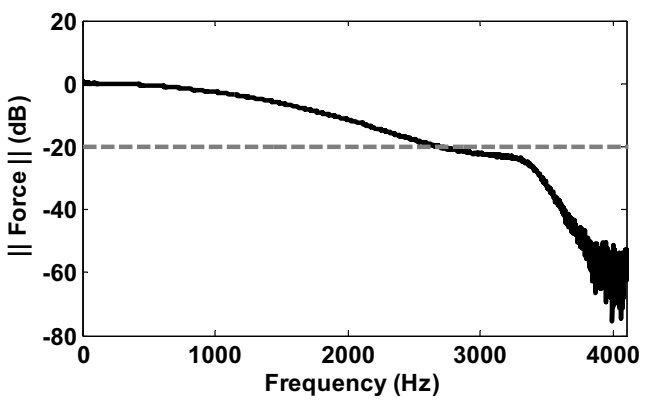

Fig. 13. The magnitude of a typical force at Point 1 (_- ) as well as the level at which the force magnitude drops by $20 \mathrm{~dB}(---)$.

The response and force data are recorded by an Agilent VXI (Agilent E142A, $51.2 \mathrm{ksamples} / \mathrm{s}$ ) data acquisition system. Conventional force and exponential windows are applied to the appropriate time histories before calculating the FRF in order to reduce measurement noise and leakage effects, respectively. The force window is a rectangular window that has a value of one at the start of the time history and has a width of $10 \%$ of the total time span. The exponential window also has a value of one at the start of the time history, and the window exponentially decays to a value of 0.01 at the end of the time history. The time span of the impact tests is one second according to a sampling frequency of $8192 \mathrm{~Hz}$ with 8192 data points recorded. The sample rate of $8192 \mathrm{~Hz}$ is chosen because 1) it is a standard option available within the software (MRIT from the University of Cincinnati Structural Dynamic Research Laboratory) used for the modal impact testing, and 2) it is higher than twice the frequency at which the magnitude of the modal impact force falls below $20 \mathrm{~dB}$ of the force values at frequencies very near $0 \mathrm{~Hz}$. The magnitude (in $\mathrm{dB}$ ) of a force applied at Point 1 from a modal impact hammer with a metallic tip is shown in Fig. 13.

After the FRF training matrix is populated, the modal hammer with a metal tip is again used to impart a force on the canister at one of the 24 determined locations. The force is applied in a direction normal to the canister. During and after the impact, the responses from the triaxial accelerometer are recorded. The trained FRFs found via modal impact testing and the recorded acceleration responses are then used to passively estimate the location and amplitude of the applied force.

When implementing the shape-based force estimation technique, only the frequency span of $5-1000 \mathrm{~Hz}$ is considered when determining the differences between the estimated forces and their curve-fit lines. Similarly, only the frequency ranges around the modal frequencies less than $1000 \mathrm{~Hz}$ are considered in the amplitude-based technique. As such, the frequency ranges of $520-580 \mathrm{~Hz}$ and $840-890 \mathrm{~Hz}$ are used when finding the median force values for the amplitude-based technique where the spans of the two frequency ranges are determined empirically. Although the structure is excited at frequencies above $1000 \mathrm{~Hz}$ in later sections, a different (acrylic) hammer tip is used with the modal impact hammer, and very little energy above about $1000 \mathrm{~Hz}$ is input into the structure causing low 
Table 3

Comparison of the ability to correctly locate isolated, impulse forces for the two passive techniques

\begin{tabular}{lc}
\hline Method & Accuracy (\%) \\
\hline Shape-based & 97.8 \\
Amplitude-based & 97.2 \\
\hline
\end{tabular}

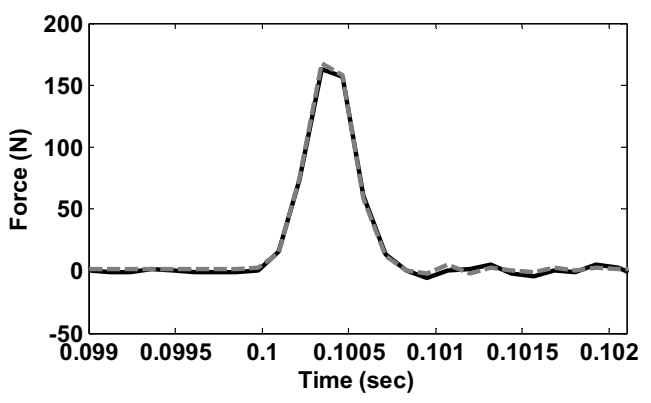

Fig. 14. Comparison of the estimated impulsive force (- - ) and actual force (-- $)$ for an impact at Point 1.

SNRs and erroneous force estimates above $1000 \mathrm{~Hz}$. The accelerometer does not accurately measure accelerations lower than approximately $5 \mathrm{~Hz}$. Therefore, the parameters of the force estimation techniques are standardized for all experimental tests if only frequencies in the $5-1000 \mathrm{~Hz}$ range are considered.

The shape-based technique for passive force estimation assumes that the force acted at one distinct point among the 24 possible input DOF. Each of these 24 force estimates is then curve-fit with a quadratic line, and the overall, normalized difference between the force estimate and the curve-fit line is calculated over the span of 5-1000 Hz. The force is assumed to act at the DOF with the lowest overall normalized difference. Because the shape-based method uses all three response measurements to estimate one force 24 times, the force is both located and quantified in the same step. Conversely, the amplitude-based force estimation technique uses multiple steps to locate and quantify the applied force.

The amplitude-based technique for force identification and quantification utilizes multiple force comparisons in iterative steps. For the chosen input-degree-of-freedom discretization of the missile casing, the first iteration of force comparisons breaks each of the six rings of four points into 2 pairs. The method for dividing the four points within each ring is arbitrary, but in this paper the pairs are based on the two lowest numbered DOF and the two highest numbered DOF within each ring. All three response measurements are used in the calculation of each force pair. For the quantification of the force acting at the last remaining DOF, a final force calculation is necessary using all three acceleration measurements to estimate the force at the determined impact point. As mentioned in the analytical section, the final force calculation for the amplitude-based approach is the same as that used in the shape-based approach for the correct impact point. An example of the final force estimate in the time domain for an impact at Point 1 is shown in Fig. 14. This result closely matches the actual force applied because no damage is caused by the impact, and the SNR for almost all frequencies is large.

The procedures for locating and quantifying isolated, impulsive forces using both the shape-based and amplitude based passive, force-estimation techniques are repeated for 20 impacts at each of the 24 possible input DOF for five different RMCs. In total, the two techniques are tested for accuracy in identifying the locations of 2400 impact forces. The accuracies of the two techniques to locate where the force acted on the structure are presented in Table 3 . The accuracies of the two techniques are relatively high when compared to the accuracies (e.g., $80 \%-100 \%$ ) of similar tests of force-identification techniques that utilize an overdetermined system of equations and/or more than one sensor [10,11]. Both techniques are successful in determining the correct impact location. It is demonstrated above that if the correct input DOF is identified, the force will be quantified with little error as shown in Fig. 14. The accuracy of the amplitudes of the force estimates was not quantitatively identified for the 2400 experimental tests, but, as in the analytical section, insight into the accuracy of the force estimation process is gained by investigating the extent to which the number of response measurements used in the calculation to estimate the force at the final input DOF changes the accuracy of the force estimate. 
a)
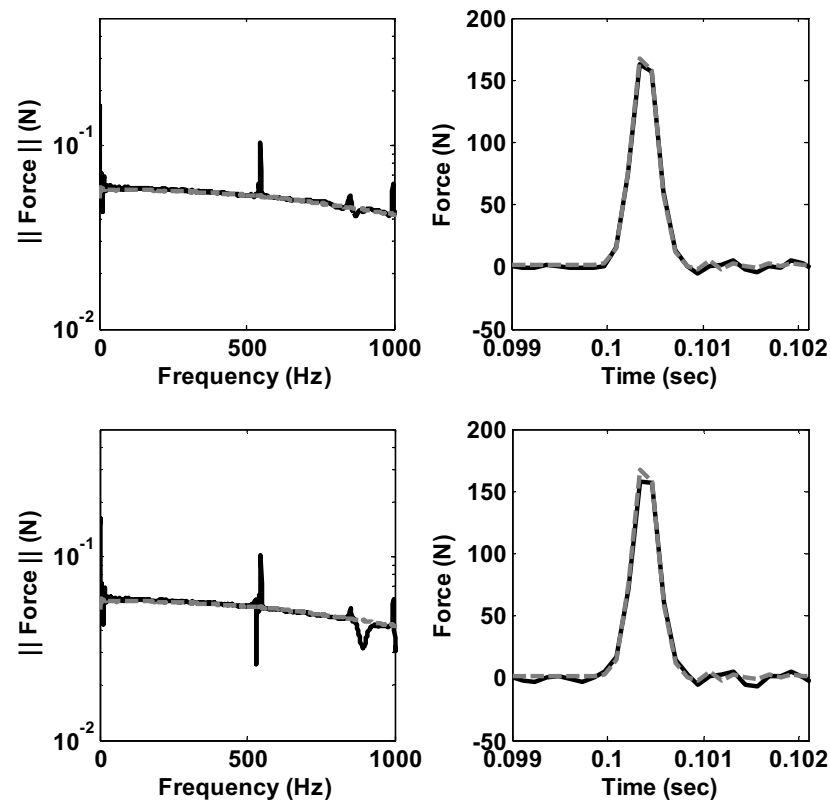

b)
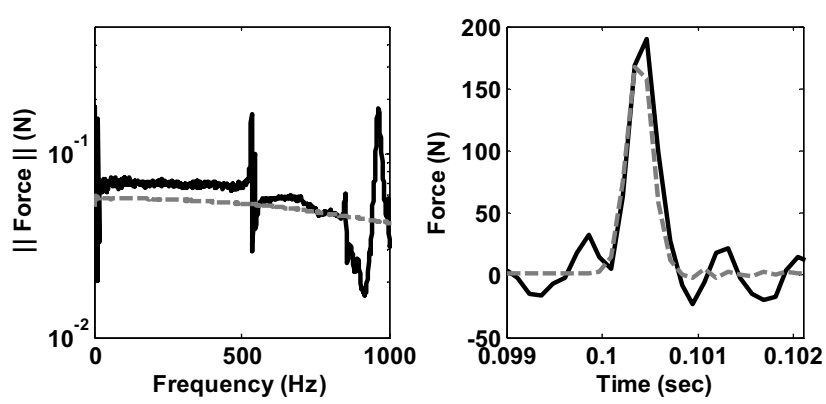

Fig. 15. Comparison of estimated force (_- ) and actual force (---) when a) three, b) two, and 3) one response measurement(s) is(are) included in the system of equations.

The number of response measurements included in Eq. (1) when quantifying the force at the final forcing DOF is varied in order to determine how the accuracy of the force estimate is dependent on the degree to which Eq. (1) is overdetermined. Because a triaxial accelerometer is used to capture the structural responses of the RMC, one, two, or three response measurement(s) can be included in the force estimate calculation. The force estimate for the force at Point 1 previously described is shown in Fig. 15 when the number of response measurements in Eq. (1) is varied. For simplicity, when one response measurement is used, the $X$-direction data are utilized, and when two response measurements are used, the $X$ - and $Y$-direction data are utilized.

Because the response measurements had little noise corruption, the difference in the accuracy of the force estimate when three or two response measurements are included in Eq. (1) is small. However, when only one response measurement is used in Eq. (1), the accuracy of the force estimate greatly reduces. This direct relationship between the number of response measurements and the accuracy of the force estimate necessitates that two response measurements, at a minimum, be available in a passive SHM system for the RMC.

\subsection{Non-impulsive impacts}

In previous force identification sections, only isolated, impulsive forces are investigated. In reality, the impact forces acting on the rocket motor casing may not be impulsive. For example, if a tool is dropped on a missile 
Accuracies of the two force estimation techniques for identifying "random" forces

\begin{tabular}{lc}
\hline Method & Accuracy (\%) \\
\hline Shape-based & 93.9 \\
Amplitude-based & 98.7 \\
\hline
\end{tabular}
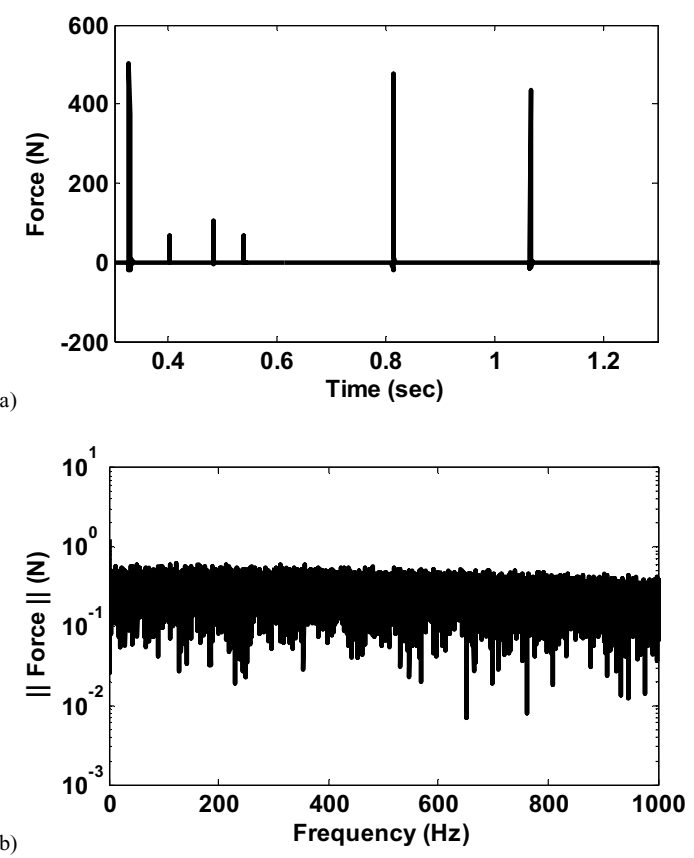

Fig. 16. An example of a "random" impact force in the a) time and b) frequency domains.

during maintenance operations, the tool may impulsively impact the casing, rebound, and again fall onto the casing. Non-impulsive forces are investigated in this section, and the forces are assumed to act at one location.

The data analyzed in this section is acquired by a National Instruments USB-9233 (50 ksamples/s) data acquisition system. The sampling frequency is $5000 \mathrm{~Hz}$, the number of points collected for each impact test is 16,384, and an acrylic hammer tip is used with the modal impact hammer instead of a metal tip. The frequency at which the force magnitude falls $20 \mathrm{~dB}$ is approximately $1000 \mathrm{~Hz}$ as discussed earlier. All other aspects of the experimental test set-up remain the same as those for the impulsive impact tests.

To further investigate the robustness of the two force-estimation techniques, "random," impulsive forcing functions are considered. An example of the type of forcing function considered "random" for this investigation is shown in Fig. 16. The magnitude of the force in the frequency domain, Fig. 16b, still possesses the global roll-off characteristic of an impulsive force. Because the roll-off characteristic is evident for non-impulsive impacts, both the shape-based technique and the amplitude base technique perform well, Table 4. A total of 540 "random" impacts were tested, and although this sample size is small compared to the impulsive impact tests, the relative accuracies of the two techniques is evident. The accuracy of the amplitude-based technique is superior to the accuracy of the shape-based technique for locating random forces because the amplitude-based technique makes no assumption about the magnitude shape or type of force applied and only compares median force values.

\subsection{Impulsive impacts acting between input DOF}

The RMC used in previous experimental tests was discretized into 24 possible input locations (force acts radially), but a force could also act on the casing at a location other than the specified 24 input DOF. This section investigates 
Table 5

Accuracies of the two force-estimation techniques to identify impacts at locations other than the trained input DOF

\begin{tabular}{llcccccc}
\hline & & \multicolumn{5}{c}{ Distances (mm) away from DOF 4 toward DOF 10 } \\
\cline { 3 - 7 } & & 0 & 6.35 & 12.7 & 25.4 & 38.1 & 50.8 \\
\hline \multirow{2}{*}{ Technique } & Shape-based & $100 \%$ & $100 \%$ & $100 \%$ & $100 \%$ & $90 \%$ & $0 \%$ \\
& Amplitude-based & $100 \%$ & $100 \%$ & $100 \%$ & $100 \%$ & $0 \%$ & $0 \%$ \\
\hline & & \multicolumn{5}{c}{ Distances (in) away from DOF 4 toward DOF 5 } \\
\cline { 3 - 7 } & & 0.00 & 6.35 & 12.7 & 25.4 & 38.1 & 50.8 \\
\hline \multirow{2}{*}{ Technique } & Shape-based & $100 \%$ & $100 \%$ & $100 \%$ & $100 \%$ & $100 \%$ & $100 \%$ \\
& Amplitude-based & $100 \%$ & $100 \%$ & $100 \%$ & $100 \%$ & $100 \%$ & $100 \%$ \\
\hline
\end{tabular}

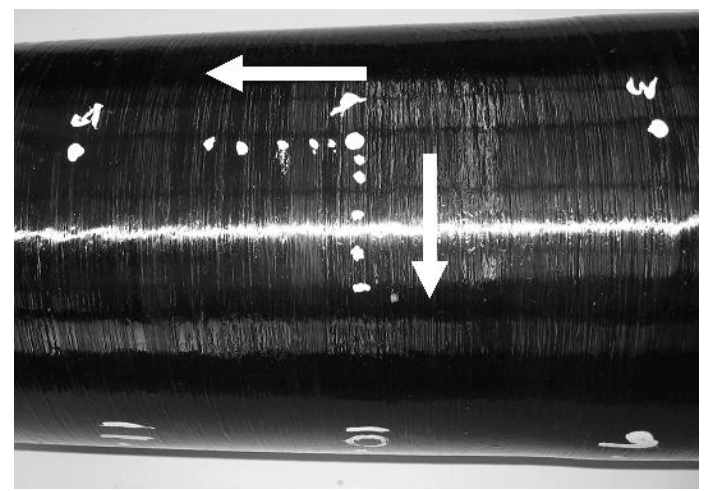

Fig. 17. The impact location was varied away from the trained input DOF in both the radial and axial directions.

the ability of the two force-estimation techniques to correctly identify the nearest input DOF for forces that do not act exactly at one of the 24 possible points. A modal impact hammer with an acrylic tip is used to impulsively impact the RMC at a specified and trained input DOF and at varying distances away from the input DOF. The input force is applied 6.35, 12.7, 25.4, 38.1, and 50.8 millimeters away from the input DOF in both the circumferential and axial directions as shown in Fig. 17.

Ten impacts are applied at the trained input DOF as well as each of the 10 points at varying distances from the input DOF. Although this is a small sample size of tests at each impact location, the relative performance of the techniques as a function of the impact location is clear. The accuracies of the two techniques to identify the nearest input DOF for the different impact location are shown in Table 5. It is evident that the ability of the force-estimation techniques to correctly identify the nearest input DOF is a function of the distance of the actual impact location from the trained input DOF and also the direction away from the input DOF. Both techniques perform well for impacts within $25.4 \mathrm{~mm}$ of the input DOF. For impacts more than $25.4 \mathrm{~mm}$ from the input DOF in the circumferential direction, the shape-based technique more accurately estimates the nearest input DOF than the amplitude-based technique. The distance between Point 4 and Point 10 is approximately $146 \mathrm{~mm}$, so Point 4 is always the nearest input DOF for the circumferential tests. The higher accuracy of the shape-based technique is most likely because of it global nature with respect to frequency. For the impacts not correctly located, both techniques predict that the impact DOF is DOF 5, so both techniques located the impacts within one DOF of the nearest DOF.

The impacts that varied from the trained input DOF in the axial direction are better located. The total distance between Point 4 and Point 5 is approximately $102 \mathrm{~mm}$, so the nearest DOF for all impacts is DOF 4 (or DOF 5 for the $50.8 \mathrm{~mm}$ test). One possible reason that the impacts away from the trained DOF in the axial direction are better located than the circumferential direction is the fact that an input in the axial direction still excites the structure in the same general directions as an impact at the trained DOF. Therefore, the signal ratios between the different triaxial accelerometer directions are approximately the same as if the impact occurred at the trained input DOF, but as the impact moves in the circumferential direction, the signal ratios between the different measurements change. The results of the tests away from the input DOF suggest that a finer mesh is needed in the circumferential direction than in the axial direction, and that the mesh seed size in the radial direction should be no more than $25.4 \mathrm{~mm}$. In general, 


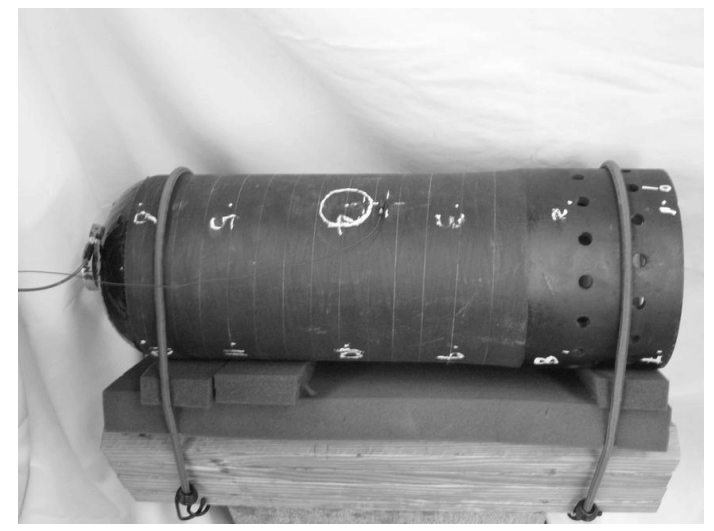

Fig. 18. Filament-wound RMC in test fixture for the drop-tower impacts.

the results suggest that the two techniques are more sensitive in certain directions or at certain input DOF for forces acting away from an input DOF. The sensitivity to impacts away from an input DOF is likely unique for every test structure and test setup.

\subsection{Damaging, impulsive impacts}

This section investigates the ability of the two aforementioned force-identification and quantification techniques to correctly estimate the amplitude of a damaging impact force. Because both techniques solve the same overdetermined system of equations for the final input DOF, the accuracies of the two techniques to estimate the force amplitude are the same. The input DOF at which the force acts is assumed to be known. The filament-wound rocket motor casings are designed to be impact resistant; therefore, an Instron Dynatup 9250HV drop tower is used to apply high-energy and damaging impacts to the RMC. For the tests involving the drop-tower, the missile casing is supported by two layers of polyurethane foam in a wooden V-block and is then strapped to the foam and wooden block with bungee cords as shown in Fig. 18. The foam is utilized in order to prevent a rattling phenomenon between the canister and the stiff wooden block, and the foam better represents the boundary conditions during normal operating conditions than placing the canister directly on the wooden block. Polyurethane foam is a common storage and packing material used for padding. The bungee cords prevent the casing from bouncing out of the foam and striking the underside of the drop-tower frame after a high energy impact.

As Fig. 19 illustrates, the filament-wound casing and wooden block are placed beneath the main cabinet of the drop tower because of size restrictions for specimens that fit in the cabinet. A tup (a rod-like structure that actually impacts the canister) with an internal force transducer strikes the canister when the carriage inside the main cabinet is released and allowed to free fall until impacting the specimen as Fig. 20 shows. The carriage inside the main cabinet of the drop tower is custom designed to be as lightweight as possible in order to better simulate low-mass objects striking the canister with high velocities (like a rock from blade-wash).

As in previous tests, a triaxial accelerometer is mounted on the nose of the missile casing during the drop-tower impacts. An Agilent VXI data acquisition system is used to acquire the data from the accelerometer and tup. The sampling frequency is $4096 \mathrm{~Hz}$ and is appropriately chosen after finding the approximate frequency at which the force falls $20 \mathrm{~dB}$ with respect to frequency. The number of points recorded is 8192 .

The FRF matrix is trained for forces at the input DOF where the tup of the drop tower hits the canister and is determined via modal impact testing. Impacts of varying energy levels are applied in order to analyze the effects of damage sustained by the missile casings. The varying impacts also allow for the identification of unaccounted-for forces including changes in the boundary condition forces resulting from the nonlinear structural properties of the foam activated by higher-energy forces compared to the forces used to train the FRF matrix. Impacts with 0.34 , $0.68,1.36,2.71,4.07,5.43,8.14,10.85$, and $13.56 \mathrm{~J}$ of energy, nominally, are applied to the canister in nine different tests. The estimated force from Eq. (1) and the curve-fit force, as described in the analytical section (without energy-balancing), are shown in Figs 21 and 22 for a $0.34 \mathrm{~J}$ impact that causes no visible damage to the RMC. A 


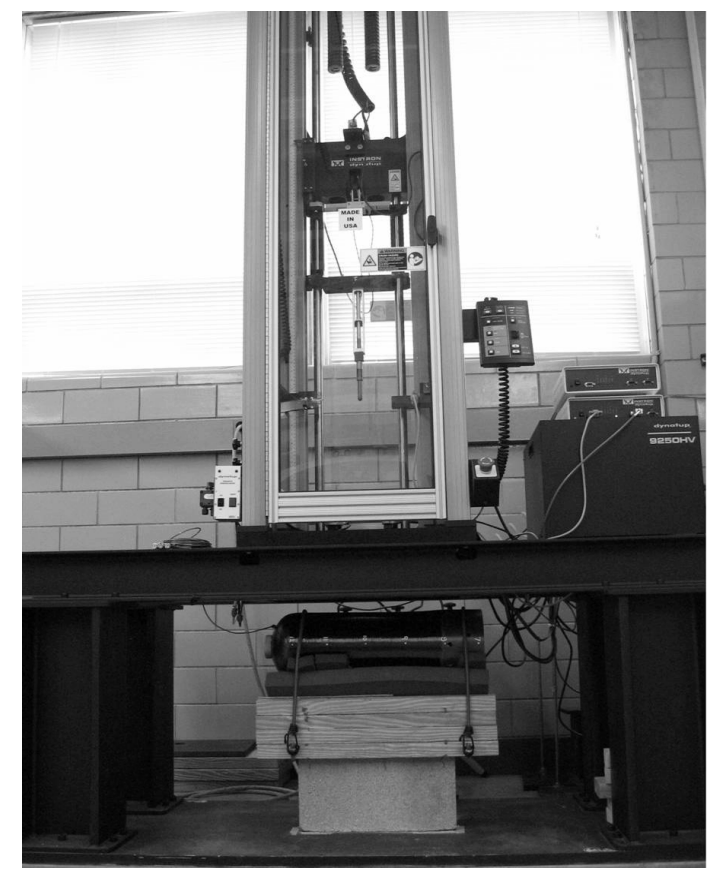

Fig. 19. The RMC and wooden block is placed below the main cabinet of the drop tower for testing damaging impacts.

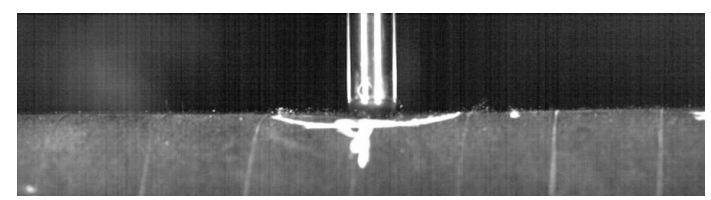

Fig. 20. A tup that is connected to the drop carriage by an aluminum extension strikes the RMC.

slight double impact occurs because the rebound of the canister after compressing the foam transpires in less time than is required by the pneumatic brakes of the drop tower to push the carriage and tup out of the way.

The actual peak force of the $0.34 \mathrm{~J}$ impact is approximately $845 \mathrm{~N}$, which is slightly higher than some of the modal-impact-hammer forces (typically between 222-667 N) identified in previous sections, and the impact caused no visible damage to the canister. However, the main lobe of the impact is approximately 2.5 times as wide as a typical impulsive force from a modal impact hammer. Accordingly, the frequency at which the magnitude of the force drops $20 \mathrm{~dB}$ is approximately $400 \mathrm{~Hz}$ (as shown in Fig. 22), which is about 2.5 times less than the corresponding frequency for a modal impact using the acrylic hammer tip. Therefore, a much larger portion of the energy of the drop-tower impact is focused at frequencies less than $400 \mathrm{~Hz}$. Low-frequency oscillations have larger deflections than high-frequency oscillations because of inertia effects, and the increased energy in the lower frequencies for the drop-tower impacts causes larger deflections of the foam supports than during modal-impact tests. As Fig. 23 shows, the stiffness of the foam varies with foam compression causing the boundary-condition forces to change from those initially modeled in the trained FRF matrix.

Because of the nonlinear properties of the polyurethane foam and other unaccounted-for forces, the ability of the force-estimation technique to accurately estimate the amplitude of the applied force is greatly hindered. As previously mentioned, the techniques are developed for and based on modeling a structure as a discrete, linear structure. The FRF matrix must also be trained while the structure is its normal operating conditions and subject to typical boundary-condition forces. The tests described in this section violate both of these assumptions. First, the composite missile casing is not a discrete, nor a linear, system. All structures have inherent nonlinearities, and high-amplitude, damaging excitations usually amplify these nonlinearities. Second, the polyurethane has nonlinear 

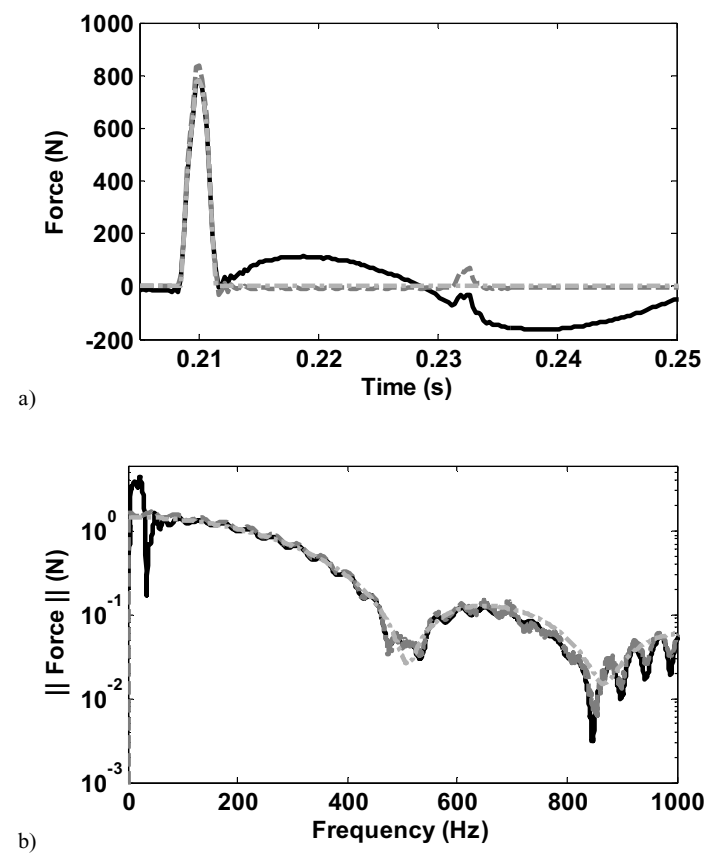

Fig. 21. The original estimate (- - , actual $(---)$, and curve-fit estimate $(-\cdot-\cdot)$ forces for a $0.34 \mathrm{~J}$ drop-tower impact in the a) time and b) frequency.

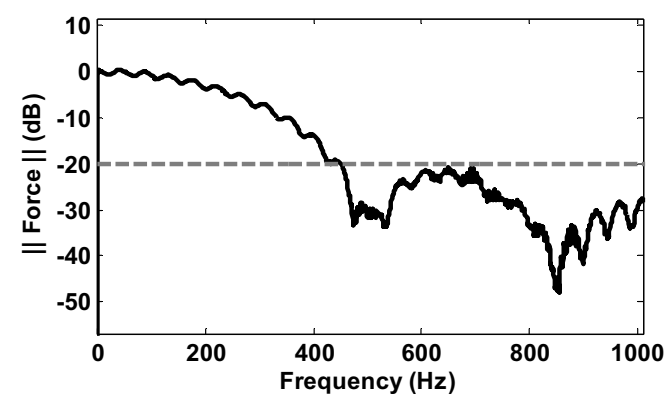

Fig. 22. The actual force (- ${ }_{-}$and the level at which it drops $20 \mathrm{~dB}(---)$ for a $0.34 \mathrm{~J}$ impact domains.

stiffness properties that vary with the amplitude and frequency of the imposed deflections [20]. The errors in the force estimate shown in Fig. 21 are due to forces that are not initially modeled in the trained FRF matrix including the nonlinear foam forces exerted on the canister during and after the drop-tower impact. Furthermore, the force estimate for the $0.34 \mathrm{~J}$ impact (Fig. 21) qualitatively indicates that the foam is the main source of the estimation errors because the force estimate largely differs from that of the actual force at frequencies lower than 50 Hertz, Fig. 21b, where no structural modes other than rigid-body modes, which are dependent on the foam properties, are present. The errors in the curve-fit force estimate caused by the differences in the rigid-body modes are also evident when comparing the actual force to the estimated force in the time domain in Fig. 21a.

The foam is also isolated as the primary source of the error in the force estimate by considering a high-amplitude impact on a RMC that is suspended by two rubber bands. The boundary conditions of the canister when suspended with rubber bands are much less influential on the structural response following a high-amplitude impact than the polyurethane foam in the wooden block. A modal impact hammer is used to apply a force with peak amplitude of over $1779 \mathrm{~N}$, nearly twice the peak force of the $0.34 \mathrm{~J}$ drop-tower impact. Similar to the $0.34 \mathrm{~J}$ drop-tower impact, the $1779 \mathrm{~N}$, peak-amplitude force causes no visible damage. However, unlike the $0.34 \mathrm{~J}$ impact, the impact when the canister is suspended is accurately predicted as shown in Fig. 24. The percent difference between the actual and 


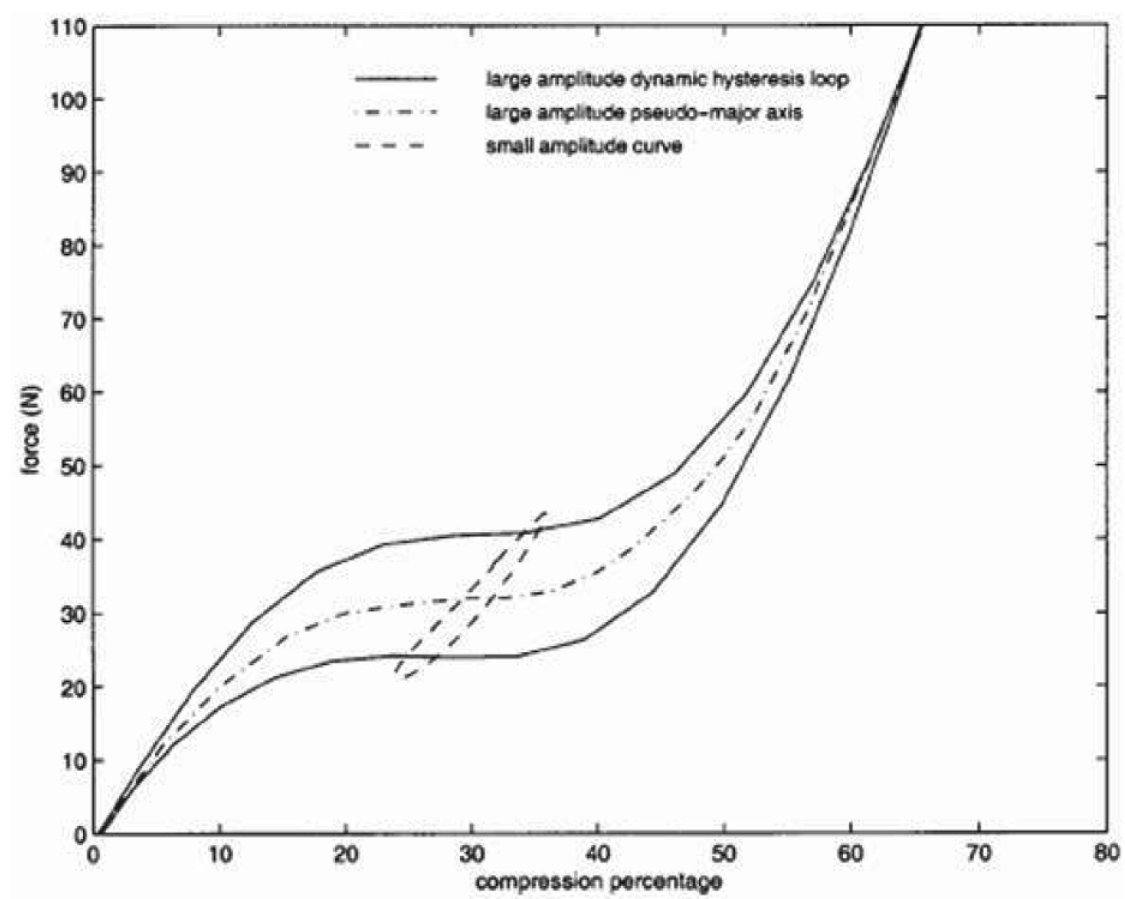

Fig. 23. Typical force/deflection curves for high and low amplitude, single frequency strain cycling of polyurethane foam (Courtesy of White et al. [20]).
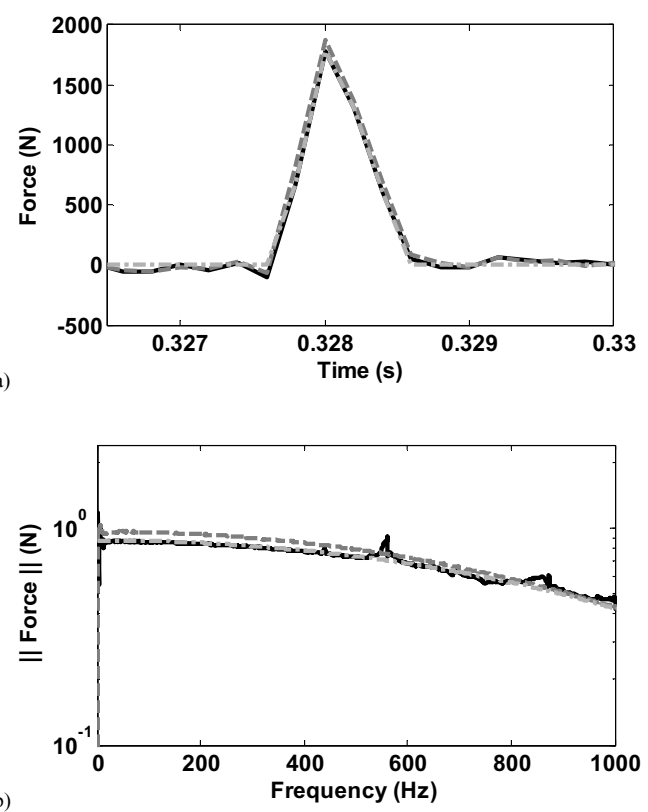

Fig. 24. Original force estimate (- - , the applied force $(---)$, and the curve-fit estimate $(-\cdot-\cdot)$ of the force for a high-amplitude impact when the canister is suspended with rubber bands in the a) time and b) frequency domains.

estimated peak force is qualitatively much less when using rubber bands as supports, Fig. 24, than when using foam, Fig. 21. The large discrepancies between the preliminary curve-fit force and actual force at low frequencies, as seen in the tests involving foam supports (Fig. 21), are not present when using the less-influential rubber-band boundary 

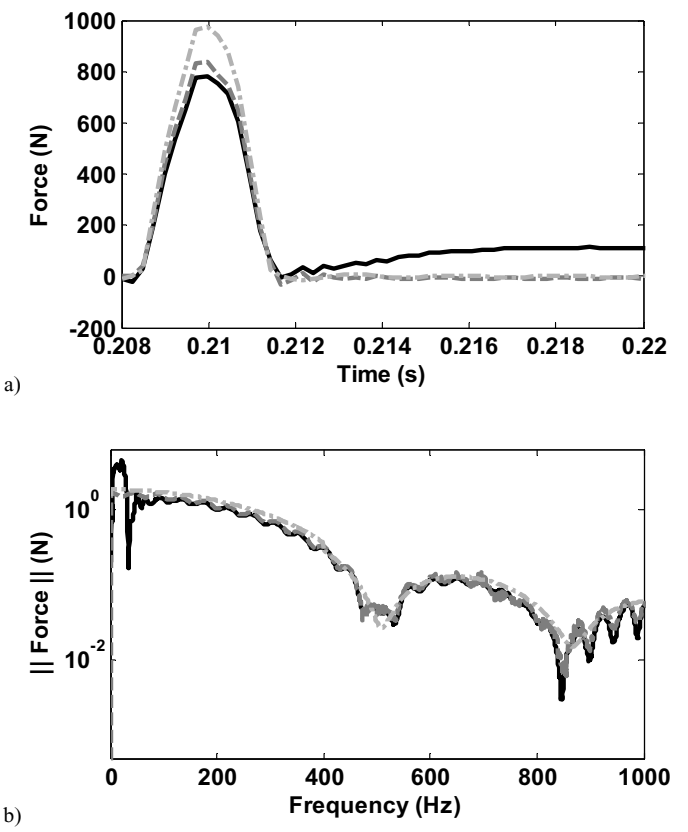

Fig. 25. Comparison of the originally estimated ( - ), actual $(---)$, and adjusted curve-fit $(-\cdot-\cdot)$ forces in the a) time and b) frequency domains.

conditions. When the results of the experiment utilizing rubber bands rather than polyurethane foam are considered, the error in the force estimate for the $0.34 \mathrm{~J}$ drop-tower impact is isolated as being caused by the nonlinearities of the polyurethane foam.

The errors of the force estimate because of the unaccounted-for forces also affect the curve-fit force estimate after equating the signal energies of the original and the preliminary curve-fit force estimates (shown in Fig. 21) as described in the analytical application. The signal energy in the frequency span of 5-400 Hz is equated between the original and the preliminary curve-fit force estimates for the non-damaging $0.34 \mathrm{~J}$ impact; the adjusted curve-fit force is shown in Fig. 25. As Fig. 25 illustrates, the signal energy of the original estimate is skewed by the frequency content below $50 \mathrm{~Hz}$ (which is frequency content that is adversely affected by the properties of the polyurethane foam), and the adjusted curve-fit force estimate over-predicts the amplitude of the applied force. The peak force of the originally estimated force is lower than the actual force for most drop-tower impacts tested in this paper, and the peak force of the adjusted curve-fit force always over-predicts the force. As Fig. 26 shows, the peak amplitude of the original force estimate more accurately predicts the actual peak force because the adjusted curve-fit estimate is greatly affected by the boundary condition forces of the polyurethane foam.

As the impact energy increases, the original estimates become less accurate because 1) the nonlinear boundarycondition forces of the foam are amplified and 2) structural damage is sustained with impacts greater than or equal to $4.07 \mathrm{~J}$. However, the results of Fig. 26 suggest that the actual peak force is bounded by the original and the adjusted curve-fit force estimates. Unfortunately, the difference between the two estimates can be significant (hundreds of Newtons), and neither estimate ensures that both the peak force in the time domain and the correct shape of the magnitude in the frequency domain are correctly estimated.

In order to increase the accuracy of the estimated force, the adverse effects of the foam boundary conditions and other forces not modeled in the trained FRF need to be eliminated. Previous discussions isolated much of the foam effects to frequencies lower than approximately $50 \mathrm{~Hz}$. In order to minimize the adverse foam effects at low frequencies, the assumption that the applied force is impulsive is revisited. As the ability of the shape-based technique to locate where a force acted has demonstrated, the magnitude of an impulsive force decreases with increasing frequency and can be accurately represented with a quadratic curve. Therefore, the magnitude estimate of the applied, impulsive force of the drop-tower experiments should follow a quadratic decay with respect to frequency. 


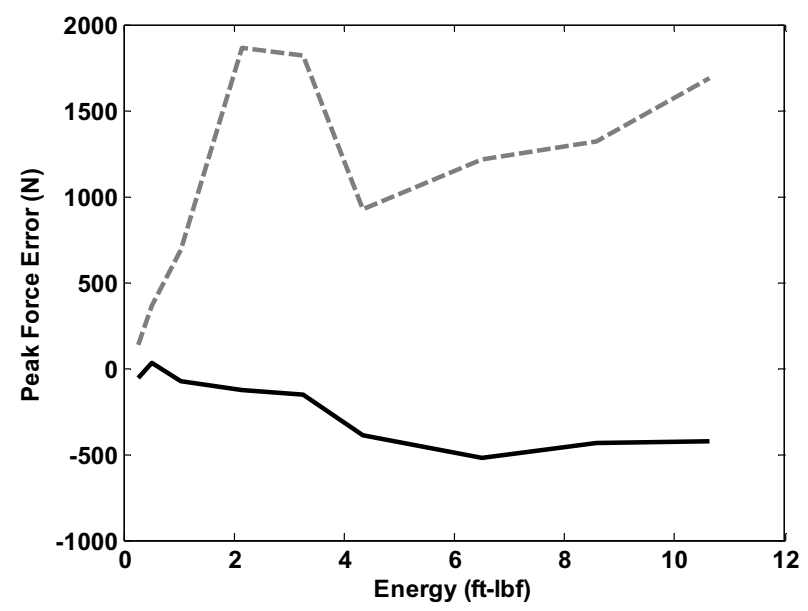

Fig. 26. Comparison of the difference between the actual peak force and the peak force of the originally estimated ( $\longrightarrow$ ) and adjusted curve-fit $(---)$ forces.

The magnitude of the drop-tower force at low frequencies is adjusted to follow a quadratic, curve-fit line according to the impulsive-force assumption. Although the majority of foam effects are below $50 \mathrm{~Hz}$, by inspecting Fig. 25, adverse effects from the foam properties are apparent up to frequencies around $100 \mathrm{~Hz}$. Consequently, the following procedure is followed to reduce the effects of the foam on the force estimate:

1. Equation (1) is used to estimate the force as previous sections have described.

2. A quadratic line is curve fit to the force estimate over the frequency range of $100-350 \mathrm{~Hz}$ in order to avoid the frequency range most affected by the foam and zero-crossings of the magnitude estimate.

3. The determined quadratic line is extrapolated toward $0 \mathrm{~Hz}$, and the magnitude of the originally estimated force is adjusted to follow the curve-fit line. Although the magnitude of the force is adjusted, the phase of the force remains unchanged.

The force estimate for a $0.34 \mathrm{~J}$ drop-tower impact after applying the above procedure is shown in Fig. 27. The curve-fitting process causes a discontinuity in the force estimate in the frequency domain at $100 \mathrm{~Hz}$. A discontinuity in the frequency domain introduces a sinusoidal-type of signal in the time domain as evident near the main pulse of the forcing function in Fig. 27a. The fluctuation in the frequency domain that is caused by the double impact of the tup is lost in the curve-fit portion of the force in the frequency domain. However, in the frequency ranges where the double impact information is lost, the foam effects erroneously dominated the original force estimate, and for an impulsive impact, as assumed, no double impact would be present.

The force estimate after the foam effects are minimized is used in an energy balance with the time-domain, curve-fit force of the original force estimate. Although the peak amplitude of the adjusted force estimate after minimizing the foam effects and equating the signal energy only slightly better matches the actual force for a $0.34 \mathrm{~J}$ impact, as shown in Fig. 28, the estimate outside of the main lobe of the force better matches that of the actual. The increased accuracy of the adjusted force estimate is evident in the frequency domain also, especially at low frequencies.

The peak force error between the original estimate, the adjusted estimate without removing the foam effects, and the adjusted estimate with foam effects removed is shown in Fig. 29. It is clear that by removing the effects of the foam and balancing the signal energy, the amplitude of the applied force estimate is greatly improved, especially for impacts with higher energy levels.

\section{Conclusions}

This paper presents two minimal sensing, passive force identification and quantification techniques. Although indirect force estimation is a well-studied topic, the literature has not focused on locating and quantifying forces 

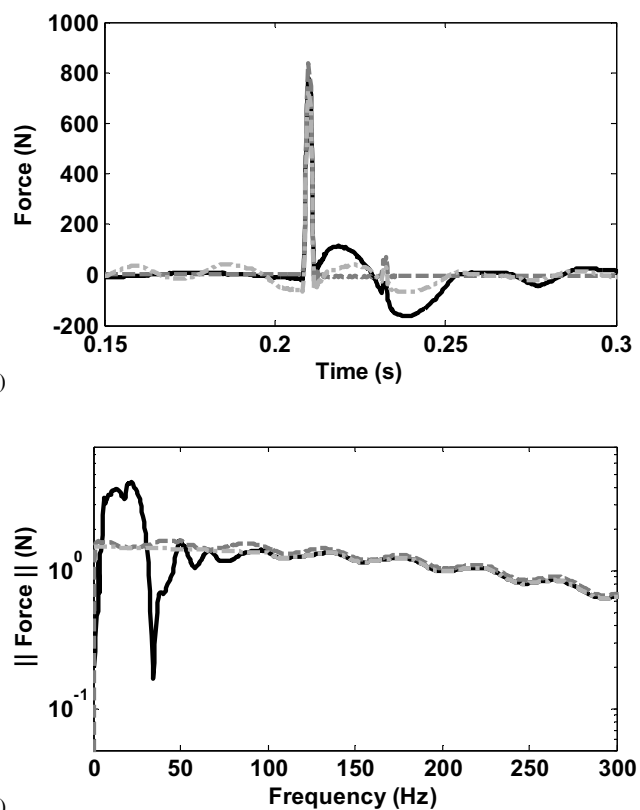

Fig. 27. Comparison of originally estimated force (- - , actual force $(---)$, and original force with the foam effects minimized $(-\cdot-\cdot)$ in the a) time and b) frequency domains.
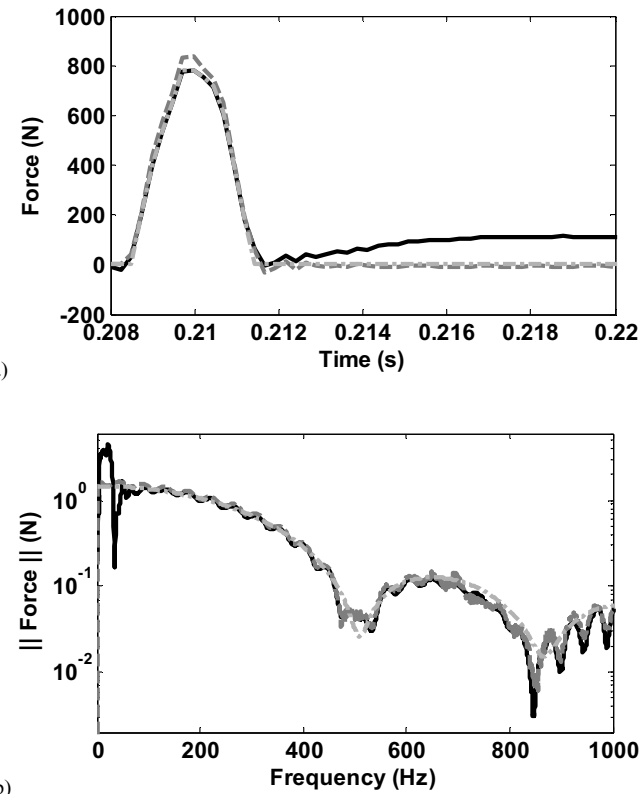

Fig. 28. The adjusted, curve-fit force $(-\cdot-\cdot)$ better matches the value and shape of the actual force $(---)$ than the original estimate $(-)$ in both the a) time and b) frequency domains.

acting on a structure with multiple input locations using only one multi-directional sensor when the structure becomes damaged due to the impact. Both force estimation techniques investigated in this paper are applied in the frequency domain. The first technique discussed is a shaped-based technique applied previously. The second method is new and is based on the amplitude of the estimated force. The shape-based technique relies on the assumption that the applied force is impulsive, but the amplitude-based technique requires no such assumptions. The shape-based 


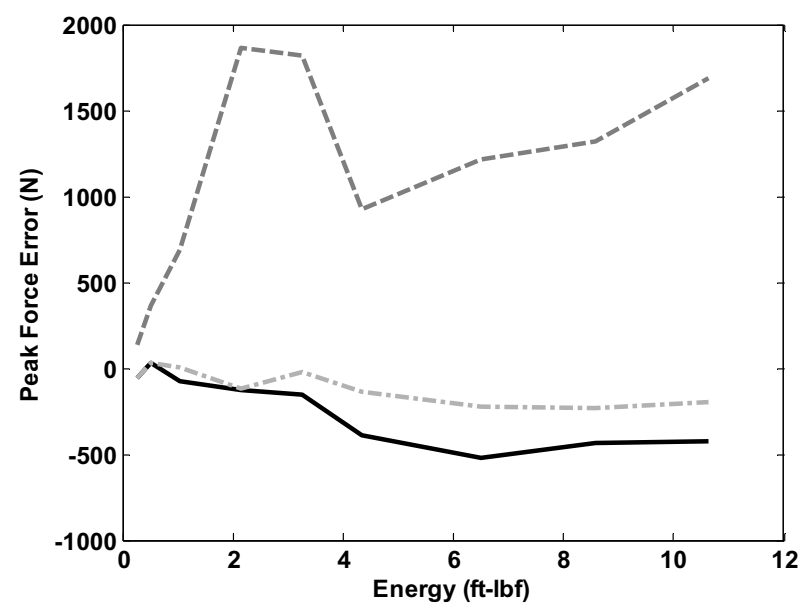

Fig. 29. Peak force error for the originally estimated force (- - ), the adjusted, curve-fit force without eliminating the foam effects (-- -), and the adjusted, curve-fit force after minimizing the effects of foam $(-\cdot-\cdot)$.

technique is a global technique, i.e., it analyzes the shape of the force magnitudes over a broad range of frequencies. Conversely, the amplitude-based technique finds the median force value for a frequency region consisting of multiple, narrow frequency spans. Both force-estimation techniques reduce an underdetermined system of equations into many overdetermined systems of equations in order to locate and quantify the applied force.

The work completed in this paper yields the following conclusions:

1. The analytical application proved that although the FRF matrix is more ill-conditioned near resonant frequencies, the low SNRs for frequencies other than those near resonant frequencies cause more errors in the force estimate than the ill-conditioning of the FRF matrix. Therefore, it is advantageous to focus on frequencies near the resonant frequencies of the structure when implementing the amplitude-based technique, which is a local technique, because the force estimates will be more accurate at these frequencies.

2. When estimating forces that damage the structure or that act on a damaged structure, the processes of curvefitting the estimated force in the time or frequency domain and ensuring that the signal energy of the original estimate matches that of the curve-fit force increase the accuracy of the estimated force. In the experimental tests that estimate damaging impacts, the adverse effects of the force estimates due to the nonlinear properties of the polyurethane foam and other unaccounted-for forces are minimized before equating the signal energies of the original and the curve-fit forces. The foam effects are minimized by revisiting the assumption that the applied force is impulsive, and that the magnitude of an impulsive force has a smooth roll-off in the frequency domain with increasing frequency.

3. The accuracy of the estimated force is directly proportional to the number of response measurements included in the equations of motion. For the experimental application involving the filament-wound RMC, at least two response measurements are necessary to achieve relatively accurate force estimates.

4. The two force-estimation techniques are able to identify the location of over $97 \%$ of 2400 impulsive impacts acting at a designated input DOF correctly.

5. Although the shape-based technique was developed to identify impulsive impacts, both force-estimation techniques are able to locate the input DOF for non-impulsive forces. The accuracies for both techniques for the small sample size tested is at least $93 \%$, and the amplitude-based technique performs slightly better than the shape-based technique because no assumptions about the applied force are required.

6. The techniques are able to better identify the nearest input DOF for forces that do not act at a trained input DOF if the impact acts away from the input DOF in the axial direction rather than the radial direction. All impacts at distances up to $50.8 \mathrm{~mm}$ from an input DOF in the axial direction are correctly identified, but impact locations are incorrectly identified for impacts acting at distances over $25.4 \mathrm{~mm}$ from the input DOF in the radial direction. The results of this study suggest that the discretization mesh can be coarser in the axial than the radial direction. 
The experimental results suggest that in certain instances the shape-based technique better locates and quantifies the applied force than the amplitude-based technique (e.g. when the impact does not act at a specified DOF), but in some instances the opposite is true; the amplitude-based technique performs better (e.g., when identifying nonimpulsive impacts). Therefore, it is conceivable that a force estimation technique that incorporates both the shapeand amplitude-based techniques may provide the most robust performance in passively identifying and quantifying external forces for RMCs. The integration of the two techniques is not considered in this paper, but the motivation to combine the benefits of the two methods has been established.

\section{Acknowledgments}

The authors would like to thank Glen Kiser with Rolls-Royce for his support of this research.

\section{References}

[1] K.K. Stevens, Force identification problems-an overview, in Proceedings of the SEM Spring Conference on Experimental Mechanics, Houston, Texas, 1987, 838-844.

[2] G. Strang, Linear Algebra and Its Applications, 4th Ed.: Brooks Cole, 2005.

[3] A.D. Steltzner and D.C. Kammer, Input force estimation using an inverse structural filter, in Proceedings of the 17th International Modal Analysis Conference, IMAC, vol. 1. Kissimmee, FL, 1999, 954-960.

[4] E. Jacquelin, A. Bennani and P. Hamelin, Force reconstruction: analysis and regularization of a deconvolution problem, Journal of Sound and Vibration 265 (2003), 81-107.

[5] D.C. Kammer, Input force reconstruction using a time domain technique, in American Institute of Aeronautics and Astronautics (AIAA) Dynamics Specialists Conference, Salt Lake City, UT, 1996, 21-30.

[6] T.G. Carne, R.L. Mayes and V.I. Bateman, Force reconstruction using the sum of weighted accelerations technique - max-flat procedure, in Proceeding of the 12th International Modal Analysis Conference, 1994, 1054-1062.

[7] S.E.S. Karlsson, Identification of external structural loads from measured harmonic responses, Journal of Sound and Vibration 196 (1996), 59-74.

[8] F.D. Bartlett Jr. and W.G. Flannelly, Model verification of force determination of measuring vibratory loads, Journal of American Helicopter Society 24 (1979), 10-18.

[9] J.A. Fabunmi, Effects of structural modes on vibratory force determination by the pseudoinverse technique, American Institute of Aeronautics and Astronautics Journal 24 (1985), 504-509.

[10] R.J. Hundhausen, D.E. Adams, M. Derriso, P. Kukuchek and R. Alloway, Transient loads identification for a standoff metallic thermal protection system panel, in Proceedings of the IMAC-XXIII: A Conference and Exposition on Structural Dynamics, Orlando, FL, 2005.

[11] A.A. Cardi, D.E. Adams and S. Walsh, Ceramic body armor high-velocity impact load identification acceleration response mapping, Structural Health Monitoring, An International Journal 5 (2006), 355-372.

[12] T. Roggenkamp, An Investigation of the Indirect Measurement of Broadband Force Spectra, Purdue University, West Lafayette, IN, Ph.D. Dissertation, 1992.

[13] K. Choi and F.-K. Chang, Identification of impact force and location using distrubuted sensors, AIAA Journal 34 (1996), $136-142$.

[14] X.Q. Zhu and S.S. Law, Time domain identification of moving loads on bridge deck, Journal of Vibration and Acoustics 125 (2003), 187-198.

[15] J.M. Starkey and G.L. Merrill, On the ill-conditioned nature of indirect force-measurement techniques, Journal of Modal Analysis (1989), 103-108.

[16] Y. Liu and W.S. Shepard Jr., Dynamic force identification based on enhanced least squares and total least-squares schemes in the frequency domain, Journal of Sound and Vibration 282 (2005), 37-60.

[17] E. Turco, A strategy to identify exciting forces acting on structures, International Journal for Numerical Methods in Engineering $\mathbf{6 4}$ (2005), 1483-1508.

[18] N. Stites, Minimal-Sensing Passive and Semi-Active Load and Damage Identification Techniques for Structural Components, Purdue University, West Lafayette, IN, USA, Master's Thesis 2007.

[19] G.T. Rocklin, J. Crowly and H. Vold, A comparison of H1, H2, and Hv frequency response functions, Proceedings of the 3rd International Modal Analysis Conference 1 (1985), 272-278.

[20] S.W. White, S.K. Kim, A.K. Bajaj, P. Davies, D.K. Showers and P.E. Liedtke, Experimental techniques and identification of nonlinear and viscoelastic properties of flexible polyurethane foam, Nonlinear Dynamics 22 (2000), 281-313. 

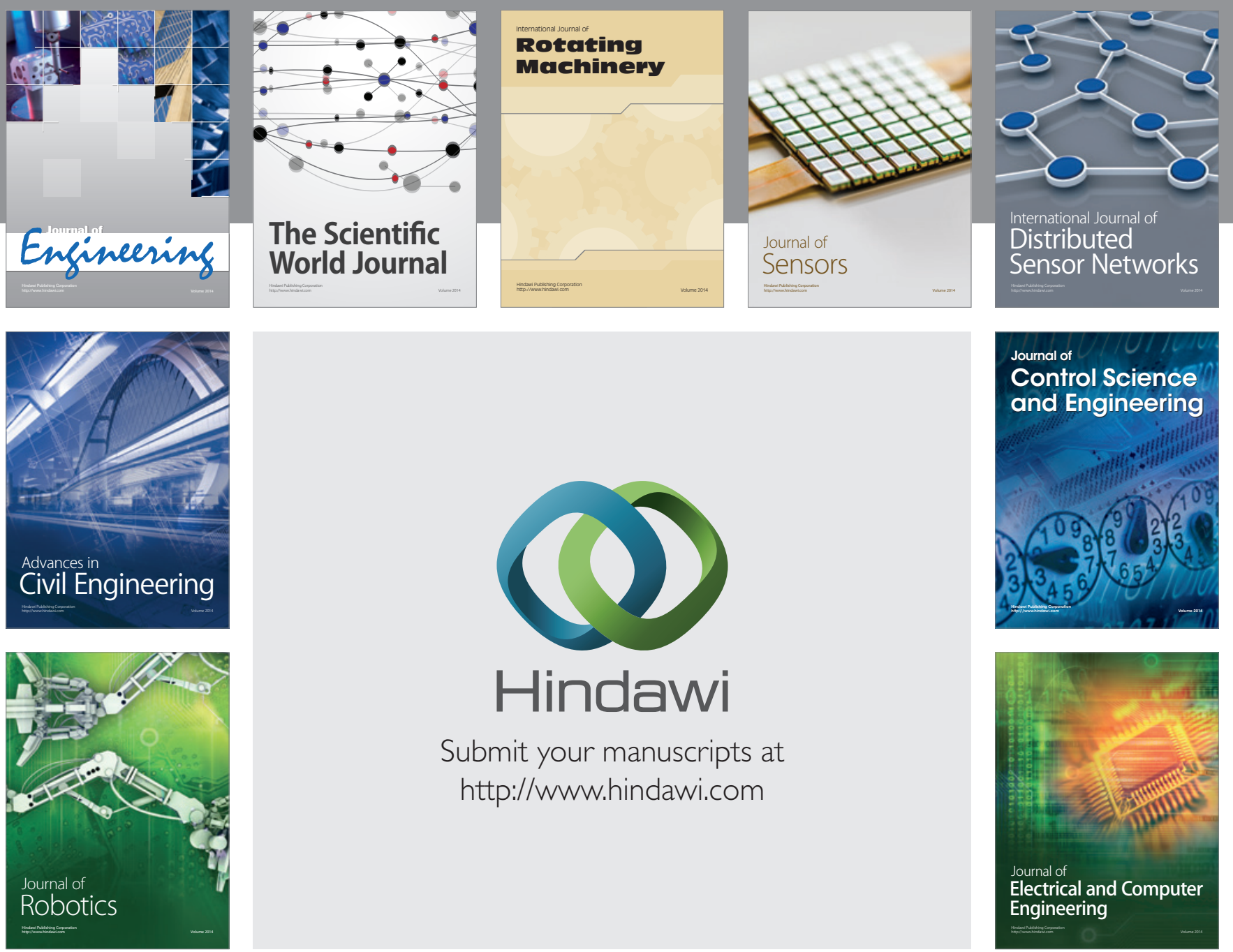

Submit your manuscripts at

http://www.hindawi.com
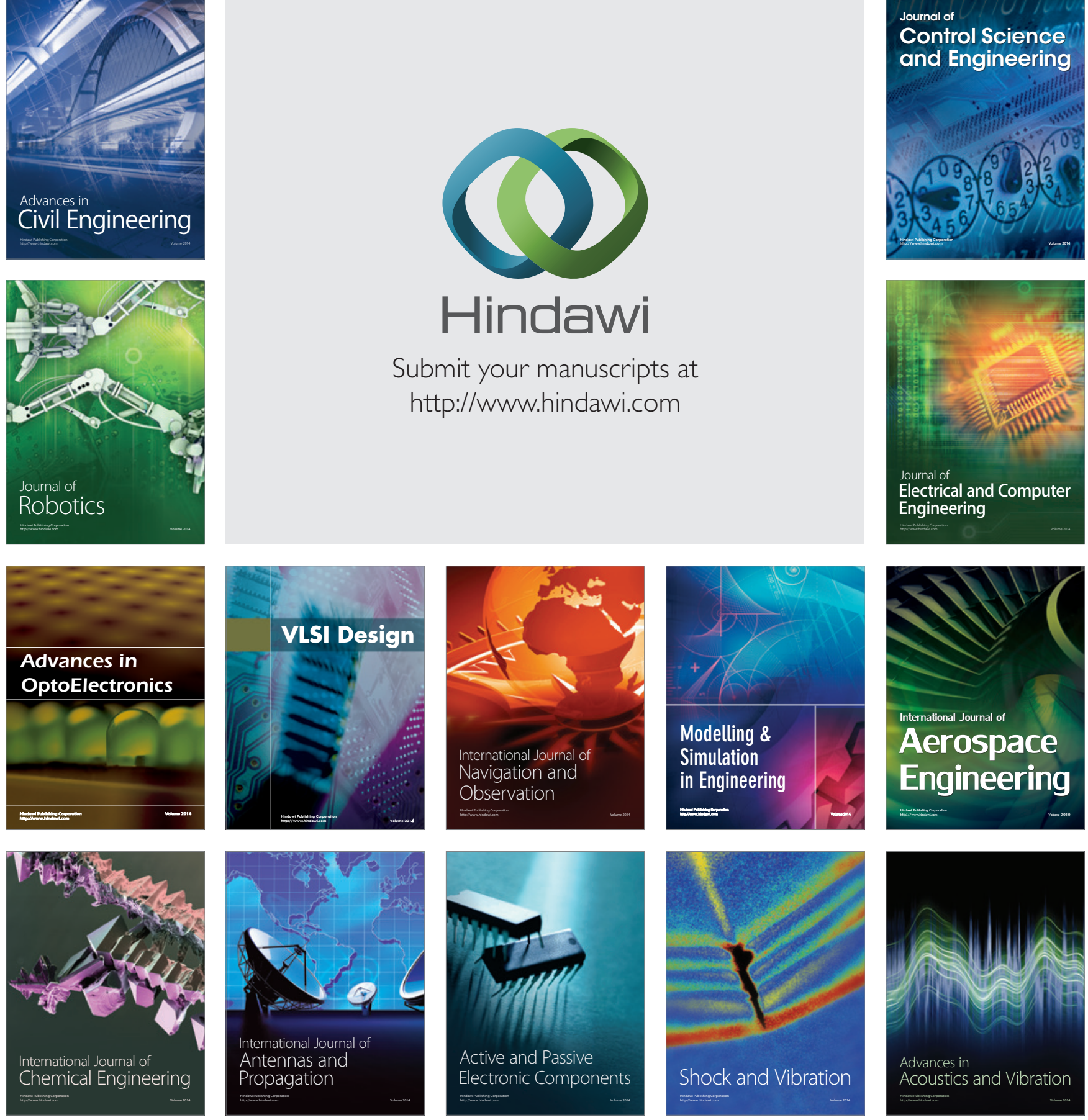